\title{
The population of OB supergiants in the starburst cluster Westerlund $1^{\star}$
}

\author{
I. Negueruela ${ }^{1}$, J. S. Clark ${ }^{2}$, and B. W. Ritchie ${ }^{2,3}$ \\ 1 Departamento de Física, Ingeniería de Sistemas y Teoría de la Señal, Universidad de Alicante, Apdo. 99, 03080 Alicante, Spain \\ e-mail: ignacio@dfists.ua.es \\ 2 Department of Physics and Astronomy, The Open University, Walton Hall, Milton Keynes MK7 6AA, UK \\ 3 IBM United Kingdom Laboratories, Hursley Park, Winchester, SO21 2JN, UK
}

Received 9 January 2010 / Accepted 8 March 2010

\begin{abstract}
Context. After leaving the main sequence, massive stars undergo complex evolution, a process that is still poorly understood. With a population of hundreds of OB stars, the starburst cluster Westerlund 1 offers an unparallelled environment to study their evolutionary tracks.

Aims. We characterise a large sample of evolved OB stars in the cluster, with the aim of determining cluster parameters and place stars in an evolutionary sequence.

Methods. We used the FORS2 instrument on the VLT to obtain intermediate-resolution spectroscopy over the range 5800-9000 of about a hundred stars selected as likely members of the cluster based on their photometry. We developed criteria for their spectral classification using only spectral features in the range observed. We discuss these criteria, useful for spectral classification of earlytype stars in the GAIA spectral region, in the appendix. Using these criteria, we obtain spectral classifications, probably accurate to one subtype, for 57 objects, most of which had no previous classification or a generic classification.

Results. We identify more than 50 objects as OB supergiants. We find almost 30 luminous early-B supergiants and a number of less luminous late-O supergiants. In addition, we find a few mid B supergiants with very high luminosity, some of them displaying signs of heavy mass loss. All these stars form a sequence compatible with theoretical evolutionary tracks. In addition, two early B supergiants also show indication of heavy mass loss and may represent the evolutionary phase immediately prior to the Wolf-Rayet stage. We investigate cluster properties using the spectral types and existing photometry. We find that the reddening law to the cluster does not deviate strongly from standard, even though extinction is quite variable, with an average value $A_{\mathrm{V}}=10.8$. Though evolutionary tracks for high-mass stars are subject to large uncertainties, our data support an age of $\gtrsim 5 \mathrm{Myr}$ and a distance $d \approx 5 \mathrm{kpc}$ for Westerlund 1 . Conclusions. The spectral types observed are compatible with a single burst of star formation (the age range is very unlikely to be $>1 \mathrm{Myr}$ ). Westerlund 1 shows its potentiality as a laboratory for massive star evolution, which can be fulfilled by detailed study of the population presented here.
\end{abstract}

Key words. stars: early-type - stars: evolution - open clusters and associations: individual: Westerlund 1

\section{Introduction}

Massive stars play a crucial role in the dynamical and chemical evolution of galaxies, providing a major source of ionising UV radiation, mechanical energy, and chemical enrichment (e.g., Massey 2003; van der Hucht et al. 2003). As they evolve from the main sequence (MS) towards the Wolf-Rayet (WR) phase, massive stars must shed most of their outer layers. Models predict that massive stars will evolve redwards at approximately constant $L_{\mathrm{bol}}$, but there is an apparent dearth of yellow supergiants with $L_{\text {bol }}$ comparable to the brightest hot stars. The lack of yellow hypergiants above a certain luminosity defines the observational Humphreys-Davidson (HD) limit, which seems to imply that stars hit some kind of instability when they reach this area of the HR diagram, and then lose mass at a formidable rate. This phase of enhanced mass loss is generally identified with

* Based on observations collected at the European Southern Observatory, Paranal, Chile (ESO 71.D-0151, 073.D-0327, 081.D0324). the luminous blue variable (LBV) stage, but there is no clear understanding of the actual evolution through the HR diagram of massive stars during the $\mathrm{H}$-shell burning phase (Humphreys \& Davidson 1994; van Genderen 2001).

There is general agreement that the WR stage corresponds to the He-core burning phase while the fact that WR stars are very hot implies that massive stars do actually loop bluewards. But there is a complex zoo of transitional objects, comprising blue supergiants (BSGs), red supergiants (RSGs), yellow hypergiants (YHGs), LBVs and OBfpe/WNVL stars, whose identification with any particular evolutionary phase is a matter of guesswork. Understanding this evolution is, however, crucial because the mass loss during this phase completely determines the contribution that the star will make to the chemistry of the ISM and even the sort of post-supernova remnant it will leave.

Unfortunately, massive stars are scarce and, as this phase is very short on evolutionary terms, examples of massive stars in transition are rare. For most of them, distances are unknown and so luminosities can be determined at best to order-of-magnitude 
accuracy, with the uncertainty feeding through to other parameters $\left(M_{*}, R_{*}\right)$. As a consequence, the difficulty in placing these objects within an evolutionary sequence is obvious.

The starburst cluster Westerlund 1 (Wd 1) may represent a unique laboratory for addressing these issues. With a mass $\sim 10^{5} M_{\odot}$ (Brandner et al. 2008; Mengel \& Tacconi-Garman 2008), it is young enough to contain a large number of OB stars, but old enough to contain stars in all the evolutionary stages. Large populations of WR stars and transitional massive stars (Negueruela \& Clark 2005; Clark et al. 2005, henceforth C05) have been identified. In this paper, we set their evolutionary context by characterising the population of moderately evolved massive stars in the cluster, i.e., objects that have started to move away from the MS, but have not yet reached the region of instability. With this information, we are able to constrain the cluster parameters and check the agreement with evolutionary tracks, which supports the idea of an (approximately) single-age population.

\section{Observations}

Observations of stars in Wd 1 were carried out on the nights of 2004 June 12th and 13th using the spectro-imager FORS2 on Unit 1 of the VLT (Antu) in three different modes: longslit, multi-object spectroscopy with masks (MXU) and multi-object spectroscopy with movable slitlets (MOS). We used grisms G1200R and G1028z, which provide the highest dispersions, to obtain intermediate-resolution spectroscopy. G1200R has a nominal dispersion of $0.38 \AA /$ pixel over the spectral range 5750-7310 ̊. G1028z has a nominal dispersion of $0.42 \AA /$ pixel over the spectral range 7730-9480 . Together, they cover most of the red and nearest infrared range.

For the longslit mode, we used a 0.3 slit to obtain the highest resolution available. Measurements of arc lines give $F W H M$ of $\sim 0.9 \AA$ with the G1200R, which results in $R$ slightly above $\sim 7000$. With the G1028z, we measure $F W H M \sim 1.3 \AA$, implying a resolution slightly below $\sim 7000$. For the multi-object modes, we used $1^{\prime \prime}$ slits $\left(10^{\prime \prime}\right.$ long in the MOS mode and of variable $<10^{\prime \prime}$ - length in the MXU mode). This results in a resolving power $R=2200$ in the red range (the exact wavelength coverage depends on the position in the CCD) and $R=2500$ in the near-IR range.

We also used the MOS mode with grism G150I to obtain low-resolution spectroscopy of stars in the field. This mode gives a resolving power $R=260$ over a range $\sim 6000-11000 \AA$ (the exact range depending again on the position within the CCD). This mode was used mainly for Wolf-Rayet stars, but other objects were also observed.

The field of view of FORS2 is $6.8 \times 6{ }^{\prime} 8$. Within the central 5'.0 $\times$ 5'.0 field of view, we selected our targets from the list of likely members of $\mathrm{C} 05$. For the external regions, targets were selected at random amongst relatively bright stars. In total, we took three MXU and one MOS mask with both G1200R and G1028z grisms, two further MXU masks with only the G1200R (these were aimed at relatively faint objects, which were expected to be OB stars near the MS and so not to have strong features in the range covered by G1028z) and three MOS masks with G150I. This resulted in $\sim 120$ stars observed with G1200R, $\sim 75$ stars observed with G1028z and 50 stars observed with G150I. More than $90 \%$ of the spectra turned out to correspond to OB stars, and hence cluster members.

In this paper, we restrict our analysis to the brightest OB members of the cluster, leaving analysis of the unevolved population of OB stars for a future work, where our FORS2 data will be combined with FLAMES and ISAAC observations. We complement our dataset with observations of a few additional bright $\mathrm{OB}$ stars which could not be observed during the FORS2 run. A few stars (W5, W7 and W52) were observed with the ESO Multi-Mode Instrument (EMMI) on the New Technology Telescope (NTT) at the La Silla Observatory (Chile) on the nights of June 5-8, 2003, using gratings \#6 and \#7, giving a coverage similar to that of the FORS observations at comparable resolution (see Clark et al. 2010, for details). Some other stars were observed with the NTT and EMMI on the nights of 15-17 February 2006. On this occasion, we used grism \#6, which covers the range 5800-8650 $\AA$ at a resolution $R \sim 1500$, though several objects are only detected from $\gtrsim 6500 \AA$.

For completeness, we include a few other objects which have been observed only in the $I$ band with the multi-object fibre spectrograph FLAMES, on the VLT. Details of those observations are reported in Ritchie et al. (2009a). The spectra cover the range 8484-9000 $\AA$, with a resolving power $R \approx 16000$.

\section{Results}

We have selected for our sample those bright stars not listed as yellow or red supergiants (C05) or Wolf-Rayet stars (Crowther et al. 2006a). The sample consists of 57 stars spectroscopically identified as luminous OB cluster members. They are listed in Table 1.

Wd 1 is too obscured to allow the acquisition of classification spectra. Even with 8-m class telescopes, moderate SNR cannot be achieved at wavelengths shorter than $5500 \AA$ with reasonable exposure times. We have been forced to rely on the data available, covering the range between $\sim 6000 \AA$ and $9000 \AA$. Criteria for classification in this range were first explored in C05, and are analysed and developed in Appendix A.

As demonstrated in the appendix (see also Appendix A of C05), the classification of supergiants of spectral types later than B2 can be achieved with rather high accuracy, because of the presence of abundant metallic lines. For earlier spectral types, classification criteria are scarcer and most of them are sensitive to both spectral type and luminosity. In most cases, by using several of them at the same time, we can narrow the spectral range for a given spectrum generally down to half a spectral subtype. This is achieved here by combining the red and $I$-band spectra.

There are three very luminous B supergiants in Wd 1, which were tentatively classified as hypergiants in C05. The I-band spectra of W33 and W42a are displayed in Fig. 1. Based on them, they can be confidently classified as $\mathrm{B} 5 \mathrm{Ia}^{(+)}$and $\mathrm{B} 9 \mathrm{Ia}^{(+)}$. $\mathrm{W} 7$ is very similar to $\mathrm{W} 33$, and was also classified $\mathrm{B} 5 \mathrm{Ia}^{(+) 1}$. These objects display weak P-Cygni profiles in the O I $8446 \AA$ line, and much stronger P-Cygni profiles in $\mathrm{H} \alpha$ (see Fig. 2). Another interesting feature in the red spectra of W7 and W33 is the C II 6578, $6582 \AA$ doublet. These lines are hardly seen in MS B-type stars, but appear weakly in B2-B5 stars of moderate luminosity and can be prominent in supergiants. Its maximum strength occurs around B3 (Walborn 1980).

Amongst Wd1 stars, the strongest $\mathrm{C}_{\mathrm{II}}$ is seen in the spectra of W70 and W71, both of which were classified in C05 as B3 Ia based on their I-band features (strong O I $8446 \AA$ line by the side of $\mathrm{Pa} 16$, presence of $\mathrm{NI}$ lines around $\mathrm{Pa} 11$ ). Another

\footnotetext{
1 Note that Clark et al. (2010) observe pulsational variability in these objects and speculate that they may induce variations of up to 1 spectral subtype
} 
I. Negueruela et al.: OB supergiants in Westerlund 1

Table 1. Sample of OB supergiants in Wd 1, with BVRI photometry from C05 (when available) and spectral types ${ }^{a}$.

\begin{tabular}{|c|c|c|c|c|c|c|c|}
\hline ID & RA (J2000) & Dec (J2000) & B & V & $R$ & $I$ & $\begin{array}{l}\text { Spectral } \\
\text { type }\end{array}$ \\
\hline W2a & 164659.71 & -455051.1 & 20.4 & 16.69 & 14.23 & 11.73 & B2 Ia \\
\hline W5 & 164702.97 & -455019.5 & 21.4 & 17.49 & 14.98 & 12.48 & $\mathrm{~B} 0.5 \mathrm{Ia}^{+}$ \\
\hline W6a & 164703.04 & -455023.6 & 22.2 & 18.41 & 15.80 & 13.16 & B0.5 Iab \\
\hline $\mathrm{W}^{2} \mathrm{~b}^{* *}$ & 164702.93 & -455022.3 & 23.6 & 20.20 & 17.91 & 15.25 & O9.5 III \\
\hline W7 & 164703.62 & -455014.2 & 20.0 & 15.57 & 12.73 & 9.99 & B5 $\mathrm{Ia}^{(+)}$ \\
\hline W8b & 164704.95 & -45 5026.7 & - & - & - & - & B1.5 Ia \\
\hline W10 & 164703.32 & -455034.7 & - & - & - & - & $\mathrm{B} 0.5 \mathrm{I}+\mathrm{OB}$ \\
\hline W11 & 164702.23 & -455047.0 & 21.2 & 17.15 & 14.52 & 11.91 & B2 Ia \\
\hline W13 & 164706.45 & -455026.0 & 21.1 & 17.19 & 14.63 & 12.06 & $\mathrm{~B} 0.5 \mathrm{Ia}^{+}+\mathrm{OB}$ \\
\hline W15 & 164706.63 & -45 5029.7 & 22.8 & 18.96 & 16.38 & 13.75 & $\mathrm{O} 9 \mathrm{Ib}$ \\
\hline $\mathrm{W} 17^{* *}$ & 164706.25 & -455049.2 & 22.7 & 18.87 & 16.19 & 13.56 & O9 Iab \\
\hline W18 & 164705.71 & -455050.5 & 21.2 & 17.32 & 14.81 & 12.27 & B0.5 Ia \\
\hline W19 & 164704.86 & -455059.1 & 22.6 & 18.22 & 15.21 & 12.37 & B1 Ia \\
\hline W21 & 164701.10 & -455113.6 & 22.5 & 18.41 & 15.56 & 12.74 & B0.5 Ia \\
\hline W23a & 164702.57 & -455108.7 & 22.1 & 17.85 & 14.91 & 12.07 & $\mathrm{~B} 2 \mathrm{Ia}+\mathrm{B} \mathrm{I} ?$ \\
\hline W24 & 164702.15 & $-4551 \quad 12.4$ & 23.0 & 18.71 & 15.96 & 13.24 & O9 Iab \\
\hline W25* & 164705.78 & -455033.3 & 21.9 & 17.85 & 15.22 & 12.61 & O9 Iab \\
\hline W27* & 164705.15 & -455041.3 & 21.5 & 17.94 & 15.35 & 12.80 & $\mathrm{O} 9 \mathrm{I}+\mathrm{OI}$ \\
\hline W28 & 164704.66 & -455038.4 & 20.9 & 16.87 & 14.26 & 11.64 & B2 Ia \\
\hline W29 & 164704.41 & -455039.8 & 22.6 & 18.66 & 16.02 & 13.38 & O9 Ib \\
\hline W30 & 164704.11 & -455039.0 & 22.4 & 18.45 & 15.80 & 13.20 & $\mathrm{O}+\mathrm{O}$ \\
\hline W31 & 164703.78 & -455040.4 & - & - & - & - & $\mathrm{B} 0 \mathrm{I}+\mathrm{OB}$ \\
\hline W33 & 164704.12 & -455048.3 & 20.0 & 15.61 & 12.78 & 10.04 & B5 $\mathrm{Ia}^{(+)}$ \\
\hline W34 & 164704.39 & -455047.2 & 22.1 & 18.15 & 15.40 & 12.69 & B0 Ia \\
\hline W35 & 164704.20 & -455053.5 & 22.7 & 18.59 & 16.00 & 13.31 & O9 Iab \\
\hline W37** & 164706.01 & -455047.4 & 22.8 & 19.11 & 16.40 & 13.65 & $\mathrm{O} 9 \mathrm{Ib}$ \\
\hline W38** & 164702.86 & -455046.0 & 23.2 & 19.10 & 16.47 & 13.81 & O9 Iab \\
\hline W41 & 164702.70 & -455056.9 & 21.3 & 17.87 & 15.39 & 12.78 & O9 Iab \\
\hline W42a & 164703.25 & -455052.1 & - & - & - & - & B9 $\mathrm{Ia}^{(+)}$ \\
\hline W43a & 164703.54 & -455057.3 & 22.8 & 18.05 & 15.22 & 12.26 & B0 Ia \\
\hline W43b & 164703.52 & -455056.5 & - & - & - & - & B1 Ia \\
\hline W43c & 164703.76 & -455058.3 & 20.4 & 18.35 & 16.18 & 13.66 & O9 Ib \\
\hline W46a & 164703.91 & -455119.5 & 23.0 & 18.55 & 15.46 & 12.46 & B1 Ia \\
\hline W46b & 164703.61 & -455120.0 & - & - & - & - & O9.5 Ib \\
\hline W49** & 164701.90 & -455031.5 & 22.6 & 18.76 & 16.30 & 13.80 & B0 Iab \\
\hline W50b ${ }^{* *}$ & 164701.17 & -455026.7 & 22.8 & 19.66 & 17.21 & 14.69 & O9 III \\
\hline W52 & 164701.84 & -455129.2 & 21.8 & 17.48 & 14.68 & 11.94 & B1.5 Ia \\
\hline W54 & 164703.06 & -455130.5 & - & - & - & - & B0.5 Iab \\
\hline W55** & 164658.40 & -455131.2 & 21.6 & 17.67 & 15.25 & 12.67 & B0 Ia \\
\hline $\mathrm{W}_{56 \mathrm{~b}^{* *}}$ & 164658.85 & -455145.8 & 22.8 & 18.88 & 16.36 & 13.76 & $\mathrm{O} 9.5 \mathrm{Ib}$ \\
\hline W57a & 164701.35 & -455145.6 & 20.7 & 16.54 & 13.83 & 11.13 & B4 Ia \\
\hline W60 & 164704.13 & -455152.1 & 22.8 & 18.50 & 15.96 & 13.28 & B0 Iab \\
\hline W61a & 164702.29 & -455141.6 & 21.2 & 17.16 & 14.62 & 12.01 & B0.5 Ia \\
\hline W61b & 164702.56 & -455141.6 & 22.7 & 18.59 & 16.00 & 13.31 & O9.5 Iab \\
\hline W62a & 164702.51 & -455137.9 & - & - & - & - & B0.5 Ib \\
\hline W63a & 164703.39 & -455157.7 & 22.6 & 18.56 & 16.20 & 13.68 & B0 Iab \\
\hline W65 $5^{* *}$ & 164703.89 & -455146.3 & 22.9 & 18.73 & 16.27 & 13.68 & O9 Ib \\
\hline W70 & 164709.36 & -455049.6 & 21.2 & 16.88 & 14.10 & 11.29 & B3 Ia \\
\hline W71 & 164708.44 & -455049.3 & 21.5 & 17.01 & 14.06 & 11.16 & B2.5 Ia \\
\hline W74 & 164707.08 & -455013.1 & - & - & - & - & O9.5 Iab \\
\hline W78 & 164701.54 & -454957.8 & 21.0 & 17.06 & 14.54 & 12.04 & B1 Ia \\
\hline W84** & 164659.03 & -455028.2 & 21.3 & 17.82 & 15.60 & 13.63 & $09.5 \mathrm{Ib}$ \\
\hline W86** & 164657.15 & -455009.9 & 22.9 & 18.76 & 16.43 & 14.00 & O9.5 Ib \\
\hline W228b & 164658.05 & -45 5301.0 & - & - & - & - & O9 Ib \\
\hline W232 & 164701.41 & -455234.9 & 21.3 & 17.53 & 15.25 & 12.85 & B0 Iab \\
\hline W238 & 164704.41 & -455227.6 & 21.4 & 17.47 & 14.98 & 12.45 & B1 Iab \\
\hline W373** & 164657.71 & -455320.1 & - & - & - & - & B0 Iab \\
\hline
\end{tabular}

Notes. ${ }^{(a)}$ Objects marked with "**" have classifications based on EMMI low-resolution (grism \#6) spectra. Their spectral types are therefore less accurate. Objects marked with "***" have classifications based only on FLAMES spectra covering 8480-8920 A. Their spectral types are tentative.

object with prominent $\mathrm{C}$ II doublet is W57a. From the ratio of O I $8446 \AA$ to $\mathrm{Pa} 16$ and the intensity of the He I lines, this object is either later or more luminous than W70 (Fig. 3). From the colours and magnitudes of these stars, the last possibility seems unlikely (all three have about the same magnitude in $I$, while W57a is less reddened). This is supported by the weaker 


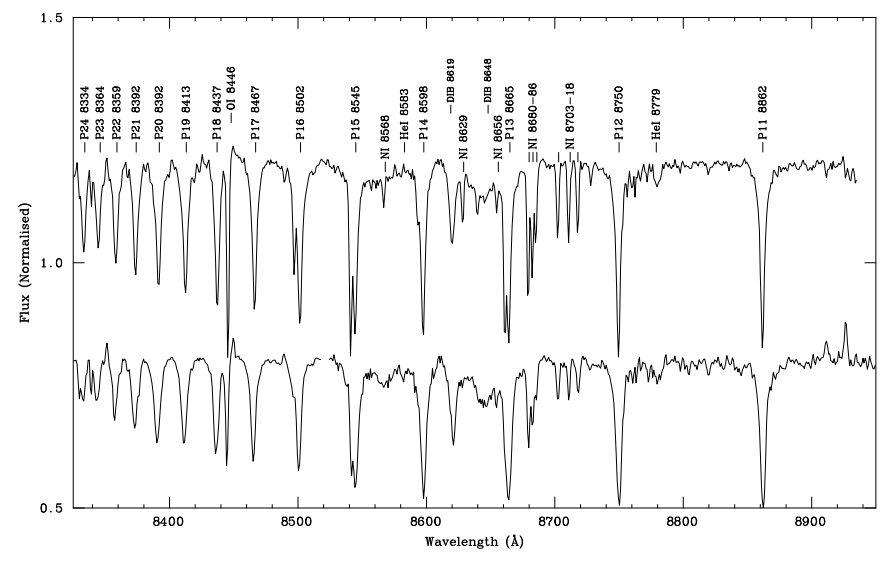

Fig. 1. I-band spectra of two of the visually brightest B supergiants in $\mathrm{Wd} 1$, tentatively classified as hypergiants in $\mathrm{C} 05$. The top spectrum corresponds to W42a, which we classify as $\mathrm{B} 9 \mathrm{Ia}^{(+)}$, based on the features presented here. Note that the Ca II triplet lines are already comparable in strength to $\mathrm{Pa} 13,15$ and 16. The bottom spectrum corresponds to W33, B5 $\mathrm{Ia}^{(+)}$. Note the prominent P-Cygni profile in O I $8446 \AA$ and the emission features at 8913 and $8927 \AA$, seen in the spectra of all the mid-B supergiants in $\mathrm{Wd} 1$.

C II doublet. W57a shows distinctive Si II 6347 and $6371 \AA$, as well as Ne I $6402 \AA$. These lines are also strong in W70, but weaker in W71. Therefore, we take B4 Ia for W57a and B3 Ia for W70. W71 is slightly earlier, and we classify it B2.5 Ia.

Then we have W2a and W28, presenting moderately strong C II 6578, $6582 \AA$ (Fig. 4) and still a conspicuous O I $7774 \AA$ triplet, a strong feature in the later supergiants. The O I $8446 \AA$ line is seen just as an inflection on the side of $\mathrm{Pa} 18$ (Fig. 3). Both display strong P-Cygni profiles in $\mathrm{H} \alpha$ and strong N II $6482 \AA$. The presence of this line is considered a signature of Ia class supergiants by Lennon (1993). We classify these objects B2 Ia. W11 is very similar and receives the same classification. W23a has a very similar I-band spectrum. It also shows N II $6482 \AA$. However, O I $7774 \AA$ is missing, and the C II doublet is very weak. These features are difficult to reconcile with any spectral type. As this star is very bright, we suspect that the spectrum may include an earlier type companion. W8b and W52 look slightly earlier than this previous group, but still have strong C II 6578, $6582 \AA$ and perhaps O I $8446 \AA$ on the wing of Pa 18 . We classify them as B1.5 Ia.

W78, still showing moderately strong C II doublet lines, has weaker O I $7774 \AA$. The absorption trough in the P-Cygni profile of $\mathrm{H} \alpha$ has almost disappeared in this object. The spectrum of W78 extends further bluewards than most others, and we can identify a very strong Si III $5740 \AA$ line, confirming a spectral type not far away from B1. Similar features are seen in W46a, $\mathrm{W} 43 \mathrm{~b}$ and W19. Though there is some evidence that these last three may be very slightly earlier, we classify all these stars as B1 Ia.

W18 and W21 show inconspicuous C II absorption lines and broader Paschen lines, though we can still see them up to Pa 20. The He I lines in the $I$-band are now almost vanished except for the line at $8780 \AA$, still moderately strong. In W21, we know that it does not show conspicuous Si III 5740, confirming that it is earlier than B1. Another similar spectrum is W61a, showing pure emission in $\mathrm{H} \alpha$. C III, if present, is very weak. We classify these three objects as B0.5 Ia.

W43a shows pure emission in $\mathrm{H} \alpha$. The Paschen lines are shallower and we can only see them up to Pa 18 . C III $8500 \AA$ is starting to contribute strongly to $\mathrm{Pa} 16$. There is no sign of $\mathrm{C}$ II or N II absorption now, but strong $\mathrm{C}$ II wind emission lines. This suggests a spectral type B0 Ia. This object is a single-lined spectroscopic binary (Ritchie et al. 2009a). W34 has a similar spectrum and receives the same classification.

W232 and W54 have broader Paschen lines, even though we see up to $\mathrm{Pa} 18$. W54 presents a neutralised $\mathrm{H} \alpha$, while W232 has it weakly in absorption. It also shows a very weak Si III $5740 \AA$ line. The lack of $\mathrm{H} \alpha$ emission likely indicates a lower mass loss and hence luminosity. We classify these objects as B0.5 Iab. Another similar spectrum is W6a, which shows variable $\mathrm{H} \alpha$ and hard X-ray emission (Clark et al. 2008), and is a likely binary (see below). As discussed in Appendix A, the strength of $\mathrm{H} \alpha$ emission correlates well with luminosity, but there are many exceptions.

W60 and W63a have similar spectra, with $\mathrm{H} \alpha$ neutralised or weakly in absorption, but show clear evidence of C III $8500 \AA$. Paschen lines are broad, and we classify these two objects as B0 Iab. Based on the overall similarity, we classify W49, for which we only have a FLAMES I-band spectrum, as B0 Iab, as well.

W61b also has $\mathrm{H} \alpha$ weakly in absorption, but has a prominent $\mathrm{C}$ III line, while Paschen lines are visible up to $\mathrm{Pa} 18$. We classify this object as O9.5 Iab, though a higher luminosity cannot be ruled out. Two other stars look like high-luminosity O-type supergiants. W74 has a very prominent C III $8500 \AA$ line, and Paschen lines up to $\mathrm{Pa} 18$. Its $\mathrm{H} \alpha$ line is weakly in absorption. We classify it as O9.5 Iab, though it also could be slightly earlier or slightly more luminous. W24 is very similar, with $\mathrm{H} \alpha$ more clearly in absorption, and its Paschen lines show that it is either less luminous or earlier than the other two. This object shows some spectral variability (Clark et al. 2010) and needs further study. We provisionally classify it as O9 I.

Similar spectra, with $\mathrm{H} \alpha$ close to being neutralised, are shown by W35 and W41. As they have stronger C III $8500 \AA$ and $\mathrm{Pa} 18$ is not clearly detected, we classify them as O9 Iab. Based on overall similarity, we also classify as O9 Iab a number of objects with lower-resolution (NTT) or incomplete (only I-band) spectra, W17, W25 and W38, though these classifications are less secure. W27 has a similar spectrum, but shows several indications of being a binary (complex $\mathrm{H} \alpha$, shallow broad Paschen lines, likely double He I $6678 \AA$ A), in accord with its high X-ray luminosity (Clark et al. 2008).

W30a displays strong variability and clear signs of binarity (see also Clark et al. 2008). Some of the spectra suggest that He II $8236 \AA$ could be present, while C III $8500 \AA$ changes between spectra. The Paschen lines are broad and shallow, typical of a spectroscopic binary. This system contains two O-type supergiants, and one of them could be earlier than $\mathrm{O} 9$.

For W31, we only have spectroscopy at one epoch, but the double-peaked structure of $\mathrm{H} \alpha$ strongly suggests that this B0 I supergiant has a companion, rendering the exact luminosity classification impossible. W10, as shown in Clark et al. (2008), is a double-lined spectroscopic binary, and therefore must contain two supergiants.

To the South of the cluster, W238 does not seem to fit exactly with the spectral sequence. It has $\mathrm{C}$ II in absorption and $\mathrm{H} \alpha$ partially neutralised, suggesting a spectral type B1 Iab or B1 Ib. However, $\lambda 8502 \AA$ looks stronger than expected, and this could also be a composite spectrum.

All the other objects have $\mathrm{H} \alpha$ in absorption and a smaller number of Paschen lines. Based on the relative strength of the C III $8500 \AA$ A feature, we classify them O9-09.5 Ib. We consider 
I. Negueruela et al.: OB supergiants in Westerlund 1
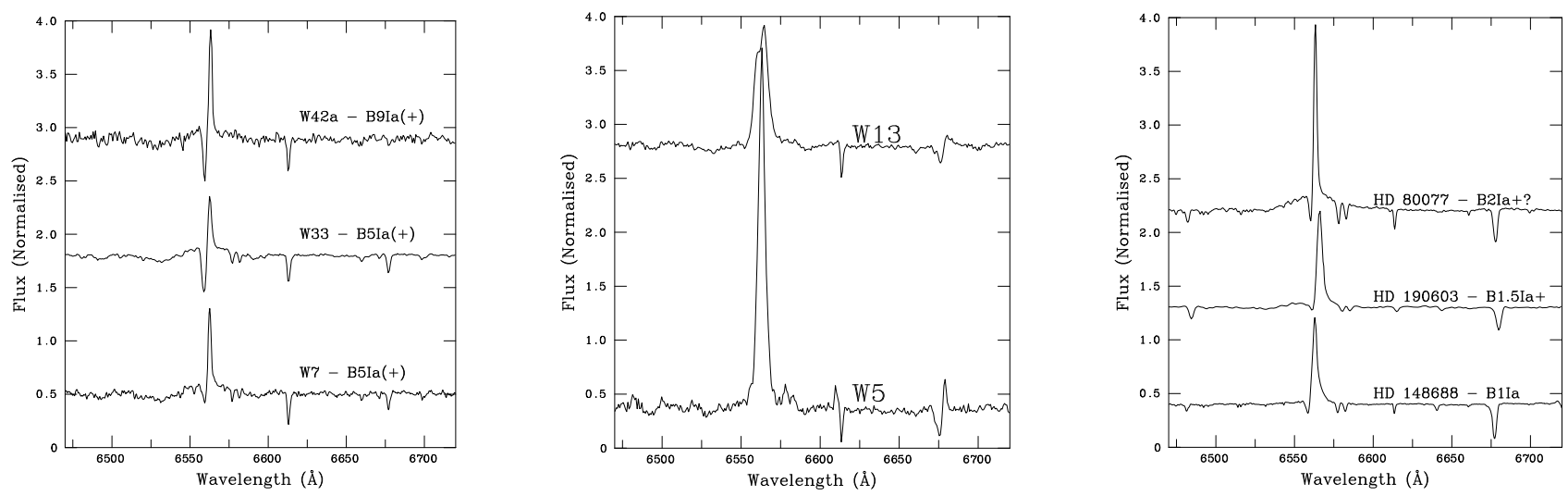

Fig. 2. $\mathrm{H} \alpha$ spectra of the most luminous B-type stars in $\mathrm{Wd} 1$ and some comparison stars. The left panel shows the spectra of the three luminous B-type objects tentatively classified as hypergiants in $\mathrm{C} 05$. The middle panel shows two objects with spectral types around $\mathrm{B} 0$ with evidence for heavy mass loss. The right panel shows, as comparison, the spectra of the hypergiant candidate HD 80077, the hypergiant HD 190603 and the supergiant HD 148688, which has the strongest emission feature amongst all the objects examined in Appendix A. All the spectra have had the continuum normalised and are offset for display.

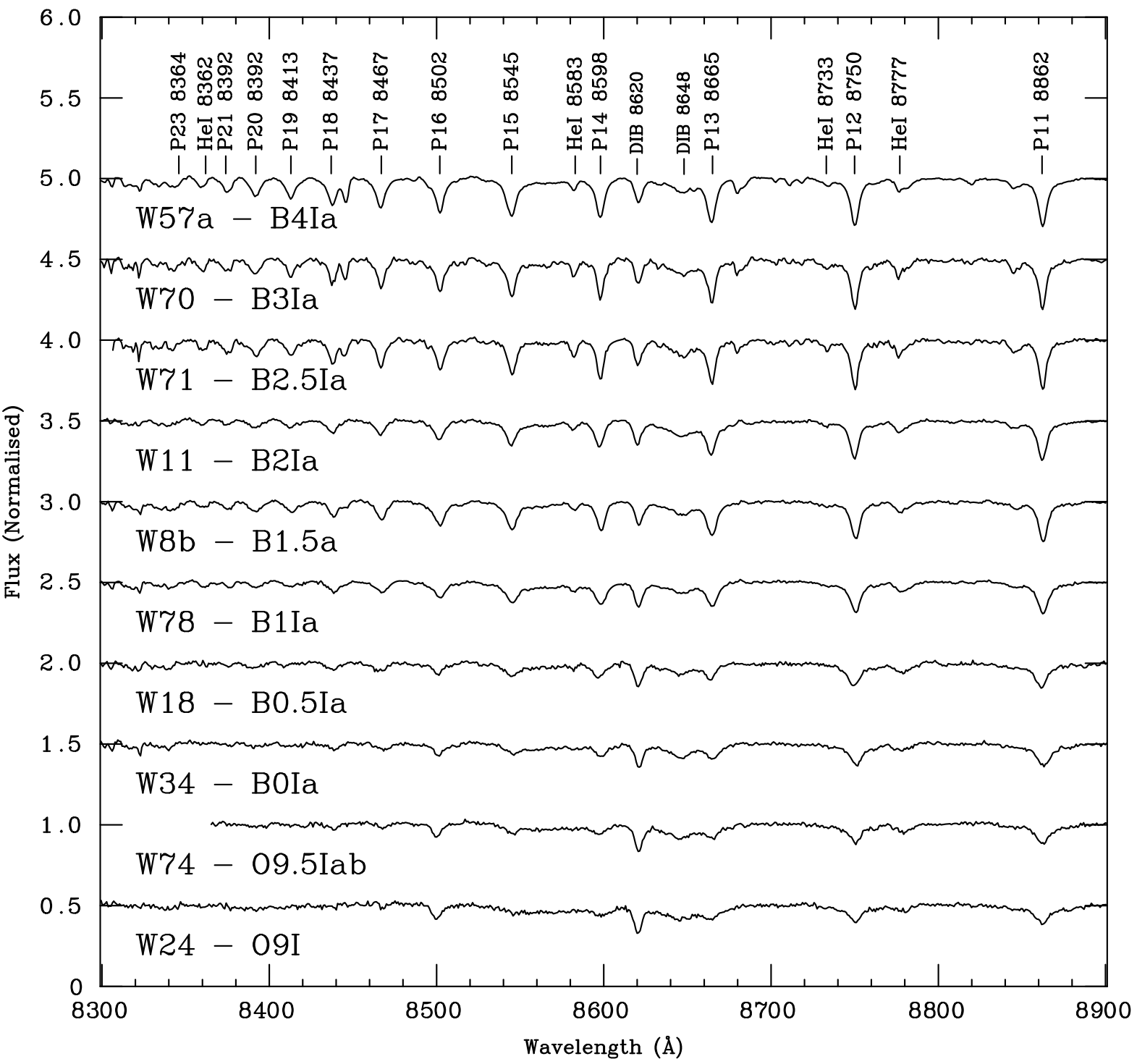

Fig. 3. Sequence of $I$-band spectra of blue supergiants in Wd 1, showing the evolution of the main features. Note the disappearance of N I features between Pa 12 and Pa 13 and O I $8446 \AA$ around B2 (compare to Fig. 1, where lines are identified) and the development of Pa 16, which shows the presence of a strong $\mathrm{C}$ III line for stars B0 and earlier. 


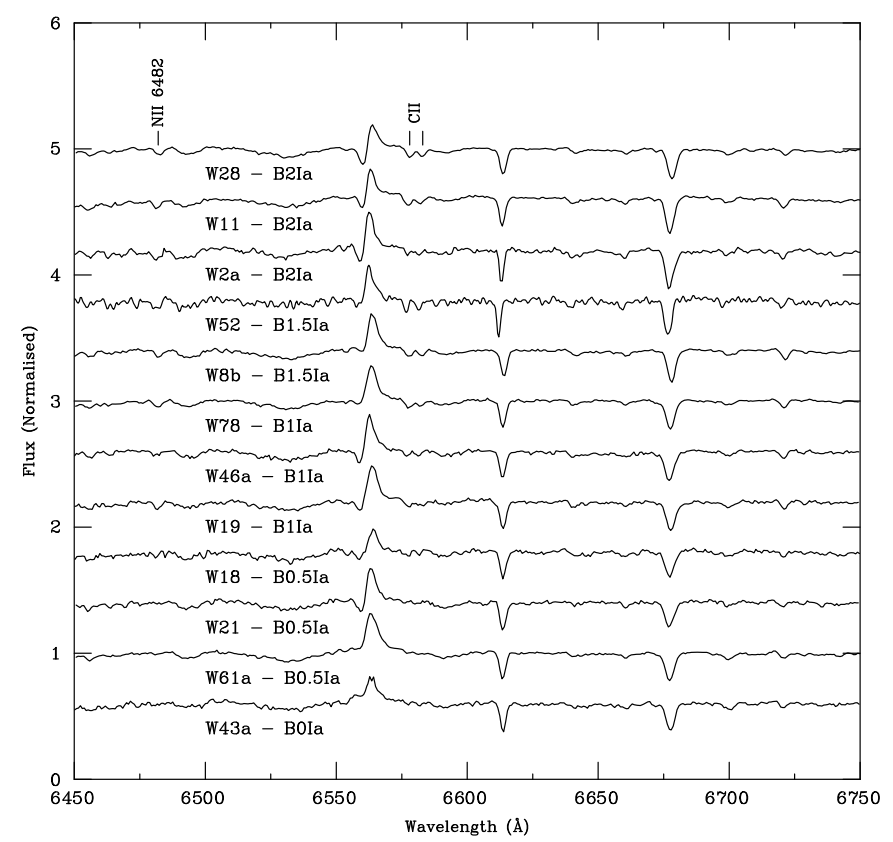

Fig. 4. Sequence of red spectra of blue supergiants in $\mathrm{Wd} 1$. The shape of $\mathrm{H} \alpha$ changes from a P-Cygni profile to pure emission around spectral type B0.5. The N II $6482 \AA$ line is only prominent in Ia supergiants and its strength peaks sharply at B2.

that having $\mathrm{H} \alpha$ in absorption to the point that it is stronger than the He I $6678 \AA$ line is the defining characteristic of luminosity class Ib. However, we caution that sky subtraction is difficult in the very crowded regions close to the cluster core, and $\mathrm{H} \alpha$ could be affected by the sky.

Taking all these factors into account, we estimate that the spectral types we derive are likely accurate to within half a subtype for B supergiants. Luminosity classes are also likely to be correct to within one subclass. Types for O stars are not so well determined, though we expect to be correct within one subtype. Luminosity classes are less reliable, as we lack proper criteria. We have to resort to line widths and shapes, which are also dependent on rotational velocity. As the rotational velocities of $\mathrm{O}$ supergiants may be relatively high, there is a degree of degeneracy between luminosity and rotation. Also, the types derived depend strongly on the ratio of the C III $8500 \AA$ line to neighbouring Paschen lines. This indicator is obviously sensitive to $\mathrm{CNO}$ anomalies. However, we do not find any spectrum where the strength of this line is openly in contradiction with any other criteria. Based on this, we believe that any $\mathrm{CNO}$ anomalies present in our stars are, at most, moderate. In the case of moderate $\mathrm{C}$ enhancement, a star classified as O9 Iab might actually be a B0 Ib with enhanced C III. In the case of moderate $\mathrm{N}$ enhancement (and, hence, C depletion), a star classified B0-0.5 Iab or Ib could be an O9 Ia with a very weak C III. None of these possible misclassifications looks too severe.

Finally, Table 1 lists the spectral types of 55 OB supergiants, and two less luminous objects, in Wd 1.

\subsection{Bright $B$ supergiants}

Two B5 Ia and one B9 Ia supergiants show characteristics that lead us to classify them as hypergiants. For a start, they are $>1$ mag brighter (after correcting for extinction, see below) than any other B-type supergiant. In addition, they show signs of heavy mass loss, such as strong P-Cygni profiles in $\mathrm{H} \alpha$ (Fig. 2) and the presence of a P-Cygni profile in O I $8446 \AA$ (Fig. 1). Clark et al. (2010) present evidence for variability in these three objects. At this stage, we do not intend their classification as hypergiants to have any implication about their evolutionary status. Most of the B5-9 I supergiants that have been subject to detailed analysis fall on tracks corresponding to stars with $\$ 25 M_{\odot}$ (McErlean et al. 1999; Markova \& Puls 2008), and hence it seems natural to find that the late B supergiants in Wd 1, most likely descendants of more massive progenitors, are more luminous. Analysis of their chemical compositions will cast light on their evolutionary status.

\subsection{Transitional objects with emission lines}

Two objects displaying strong $\mathrm{H} \alpha$ emission and $\mathrm{He}$ I emission can be classified as early B objects, W5 and W13. Their H $\alpha$ profiles can be seen in Fig. 2 (wider range spectra are displayed in Clark et al. 2008). Their I-band spectra are displayed in Fig. 5, where they are compared to the WN9 star W44 (=WR L).

W5 displays very intense $\mathrm{H} \alpha$ emission, P-Cygni profiles in He I $\lambda \lambda$ 5785, 6678 and 7065, C II wind features (not only the 7231, $7236 \AA$ doublet is in emission, but also 6578, $6583 \AA$ ) and some N II lines weakly in emission. In contrast, its $I$ band spectrum lacks any emission features and seems typical of a supergiant around B0.5 (though weak emission components in the Paschen lines may not be seen at our resolution). In view of this, we classify this object as a $\mathrm{B} 0.5 \mathrm{Ia}^{+}$star, where the hypergiant classification is due to the several indicators of heavy mass loss. However, we note that this object is intrinsically less luminous than other stars classified as B0.5 Ia. In view of this and its similarities to WN9, it is tempting to identify W5 with an evolutionary phase immediately prior to the WR stage. Again, a careful analysis of its chemical composition may provide further clues as to its evolutionary status.

W13 has a similar spectrum, but analysis is at present complicated by the fact that this object is a double-lined eclipsing binary (Bonanos 2007; Ritchie et al. 2009a). Pending a detailed analysis of the system, the data seems consistent with an emission-line component similar to W5 and an absorption-line object. Though the $\mathrm{Pa} 16$ feature in Fig. 5 is very prominent, several FLAMES spectra (cf. Ritchie et al. 2009a) show moderately strong $\mathrm{He}$ I lines, indicating that the companion is likely an early-B supergiant.

\subsection{Likely binaries}

Several spectra show evidence for binarity. In W10 we see double He I lines. In W23a, we see features incompatible with a single spectral type and variations between two epochs. W30a and W31 show evidence of mass transfer in the form of complex and (in W30a) variable $\mathrm{H} \alpha$. W30a and W27 show shallow lines, typical of double-lined binaries. W6a also shows important variations in the shape of $\mathrm{H} \alpha$ (Clark et al. 2008) and its FLAMES spectra show very shallow broad lines. The spectra of W27 have lower resolution than those of other sources, but the shape of the $\mathrm{He}$ I lines is also suggestive of two components, while $\mathrm{H} \alpha$ shows a complex emission profile. Of these, W6a, W10, W27 and W30a are hard X-ray sources (Clark et al. 2008), the latter being the most luminous and hardest detection in the cluster after the magnetar CXO J164710.2-455216 (Clark et al. 2008). W27 is also a relatively bright X-ray source $\left(L_{\mathrm{X}} \sim 10^{33} \mathrm{erg} \mathrm{s}^{-1}\right)$. 


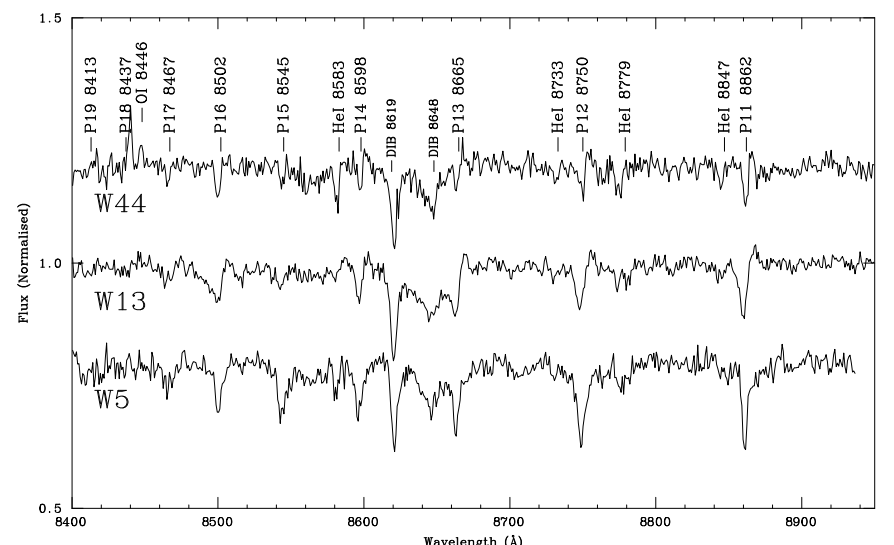

Fig. 5. $I$-band spectra of three transitional objects with emission lines. W44 (=WR L) is a late Wolf-Rayet star, presenting emission components in all Paschen lines. W13 is an eclipsing binary. One of the components is the emission-line object. W5 shows no emission lines in the $I$-band in spite of strong emission features in the red (see Fig. 2).

Considering the relative brightnesses of $\mathrm{OB}$ supergiants and MS stars, every object showing double lines must be considered to contain two supergiants. Given the relative shortness of this evolutionary phase, the presence of at least two (and likely more) objects displaying the spectra of two supergiants suggests that there is a high number of systems which actually are similarmass binaries, in good agreement with the preliminary results of a radial velocity survey, designed to determine the binary fraction in Wd 1 (Ritchie et al. 2009a).

\subsection{The less luminous objects}

In order to illustrate the characteristics of O-type stars in the $I$-band, we have included in our sample two stars with significantly fainter $I$ magnitude. These are W6b and W50b. Their spectra are displayed in Fig. 6, compared to some other objects that were observed only with FLAMES. The small spectral coverage makes all classifications somewhat uncertain, but Fig. 6 shows that a few spectral features may still provide some guidance. The main discriminant for luminosity class is the shape and depth of the Paschen lines.

The two faint stars have significantly broader Paschen lines than any star that we classify as supergiant. The limit between the wings of the lines and the continuum is difficult to define, in opposition to the sharply defined lines of the supergiants. Lacking any further criteria, we tentatively classify these two stars as luminosity class III, noting that they may well be of lower luminosity - as suggested by their dereddened magnitudes (see below). They are representative of the large population of unevolved massive stars present in $\mathrm{Wd} \mathrm{1,} \mathrm{which} \mathrm{we} \mathrm{will} \mathrm{study,}$ with a more extensive dataset, in a future paper.

\section{Discussion}

We have spectroscopically identified 55 OB supergiants in Wd 1. The sample is still very far from complete. On the one hand, the extended halo around the cluster is not fully explored. On the other hand, many stars in the cluster core, with magnitudes and colours similar to the O-type supergiants listed here, still lack spectroscopy.

The significance of this huge population has to be discussed within the context of their membership in a single cluster, $\mathrm{Wd} 1$,

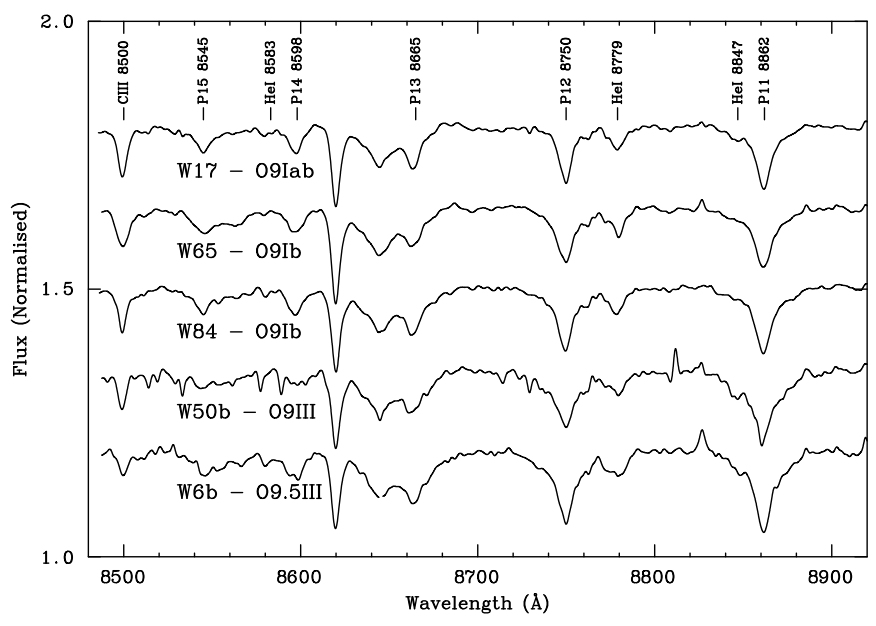

Fig. 6. Sequence of FLAMES/GIRAFFE $I$-band spectra of some of the earliest spectral types observed in our sample. The bottom two spectra have lower SNR, but the Paschen lines are clearly seen to have much broader wings than the more luminous objects and display much weaker C III $8500 \AA$ A. Note the difficulty in defining the continuum around the area of the DIBs at $8620 \AA$ and $8648 \AA$, in spite of the high resolution. The spikes seen in some spectra are badly removed sky lines.

which hosts a large population of massive stars in more advanced evolutionary stages. The cluster contains one probable active hot LBV (W9, Dougherty et al. 2010), 7 A/F hypergiants (including the cool LBV W243, Ritchie et al. 2009b), 4 very luminous RSGs (Clark et al. 2010) and a large population of WR stars (Crowther et al. 2006a). The OB supergiants provide us with the possibility to constrain the evolutionary paths of these evolved stars. Unfortunately, the parameters of Wd 1 are still poorly determined, even if significant progress has been made in recent years. The data presented here can set strong constraints on many of these parameters.

\subsection{Extinction}

The distance to the cluster is, at present, not very well constrained. Photometry is affected by very strong reddening. Analysis of the $E(B-V)$ colours suggested that the reddening could deviate from the standard law (C05), with an extinction $A_{\mathrm{V}} \approx 12$ mag. With the new accurate spectral types, we can conduct a deeper investigation of the reddening to the cluster.

We have taken all the objects with photometry in Table 1 and calculated their colour excesses, making use of the intrinsic colours for OB supergiants (Wegner 1994). Figure 7 shows the run of $E(R-I)$ against $E(V-I)$. The two colours cover a wide range, showing that the reddening is very variable across the face of the cluster. $E(V-I)$ goes from $\sim 5.0$ mag to $\sim 6.4 \mathrm{mag}$, with a single object, W84, showing rather lower reddening $E(V-I)=4.5$. The two colours are very well correlated ( $R=0.99$ for a fit without independent term). The best fit gives $E(V-I) / E(R-I)=1.97 \pm 0.01$, which compares very well with the standard reddening law (e.g., 1.95 in Rieke \& Lebofsky $1985)$. The average value is $E(V-I)=5.5 \pm 0.3(1-\sigma$ standard deviation). Similarly good fits are obtained for $E(V-I) / E(V-R)$ and $E(R-I) / E(V-R)$ (which, obviously, are not independent), giving values very close to the standard reddening law.

This suggests that the extinction is close to standard, in spite of the difficulty with the $E(B-V)$ colours. The correlation between $E(B-V)$ and $E(V-I)$ is poorer. If we force the fit to go through the origin, we obtain $E(V-I) / E(B-V)=1.31 \pm 0.01$, 


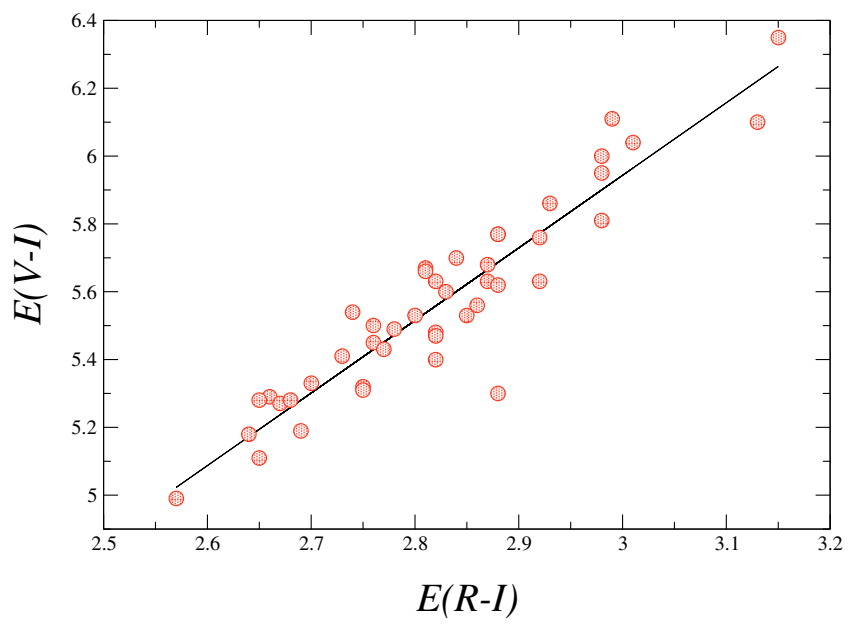

Fig. 7. Correlation between the $E(V-I)$ and $E(R-I)$ colour excesses for OB supergiants in $\mathrm{Wd} 1$. Individual excesses have been derived from the photometry listed in Table 1 and the intrinsic colour calibration.

with $R=0.97$. This value is almost identical to the average of the ratios $E(V-I) / E(B-V)$ for all the stars, $1.34 \pm 0.15$. The value is not compatible with the standard value, (e.g., 1.6 in Rieke \& Lebofsky 1985), but, because of the large dispersion in individual values, is less than $2 \sigma$ away. In view of this, we suspect that the difficulty stems from unreliable values for $B$, perhaps a zero-point offset. Even though the photometry in $\mathrm{C} 05$ was very carefully calibrated, stars in $\mathrm{Wd} 1$ are much redder than any standards used, and have very faint $B$ magnitudes. In any event, the average value directly determined from the observed $(B-V)$ colours is $E(B-V)=4.2 \pm 0.4$ (again, $1-\sigma$ standard deviation).

Another reliable indicator of interstellar extinction is the presence of Diffuse Interstellar Bands (DIBs). Unfortunately, the correlations between the Equivalent Width $(E W)$ of most bands and reddening saturates at relatively low values (e.g., Munari \& Zwitter 1997; Cox et al. 2005). Munari et al. (2008) find that the $8620 \AA \mathrm{DIB}$ is an excellent tracer of reddening, with a very tight correlation $E(B-V)=2.72( \pm 0.03) \times E W(\AA)$. As discussed in the Appendix, measurement of the intensity of this band is not easy at our resolution. However, we may expect the high number of objects to compensate for the uncertainty in individual values. Unfortunately, there is no obvious correlation between $E W(\mathrm{DIB})$ and $E(V-I)$ for our stars. Objects in $\mathrm{Wd} 1$ show an average value $E W(\mathrm{DIB})=1.00 \AA$, with a dispersion $\sigma=0.09$ and individual values ranging from 0.85 to 1.15 , with no correlation to reddening or spatial position.

If we restrict the search to objects with high-resolution FLAMES spectra, we find a similar result. The average is now $1.11 \AA$, showing that the lower resolution data tend to give lower values because of the poorer definition of the continuum. The dispersion is only $\sigma=0.08$, but still no correlation with $E(V-I)$ or location is obvious.

This suggests that this DIB is saturated, at least along this line of sight, at the reddening of $\mathrm{Wd} 1$, and the dispersion amongst $\mathrm{Wd} 1$ stars is due simply to the difficulty in determining the continuum. As discussed in the appendix, this area contains a broad shallow DIB centred on $8648 \AA$ (cf. Munari et al. 2008), which becomes compounded with some weak absorption lines. The correlation of Munari et al. (2008) would give an average $E(B-V)=3.00$, which is smaller than both the observed value and a direct extrapolation from $E(V-R)$ and $E(V-I)$ under the assumption of a standard reddening law.

There is another sharp DIB in our range, that at $6614 \AA$. Unfortunately, its correlation to $E(B-V)$ seems to be poor at high reddening values (Cox et al. 2005). Again, we find no obvious correlation to $E(V-I)$. Most stars show $E W(\mathrm{DIB})=0.60 \AA$, with standard deviation $\sigma=0.05$. As this value is comparable to the highest $E W$ (DIB) measured by Cox et al. (2005) along sightlines with high extinction, it is possible that this line is also saturated, though measurements at higher resolution should be desirable to check this hypothesis.

The only DIB that has been calibrated against $E(B-V)$ and might perhaps not be saturated at the reddening of $\mathrm{Wd} 1$ is the narrow band at $5797 \AA$. Unfortunately, this is just outside the range of our spectra. It is seen in a few of the MXU spectra, due to their variable spectral range, but the continuum cannot be defined well.

\subsection{Distribution of spectral types}

The earliest supergiants in $\mathrm{Wd} 1$ seem to have spectral types around O9. This is defined by the presence of strong C III $8500 \AA$ (while the neighbouring Paschen lines are weak or absent) and lack of He II $8238 \AA$. Even though our spectra have not been corrected for telluric absorption, humidity was very low on the night of June 13th, and the stronger He II line of earlier spectral types should be noticeable. None of the stars classified as O9 or O9.5 seems sufficiently luminous to receive a Ia luminosity class, based on their relatively broad Paschen lines and lack of detection beyond Pa 17 (but see below). W74 (where Pa 18 is weakly seen), is the most luminous O-type supergiant based on these criteria.

There are a fair number of luminous (Ia) supergiants covering the range B0.5-B2. Later spectral types become less numerous, with one each of B2.5 Ia, B3 Ia and B4 Ia, and two B5 Ia ${ }^{(+)}$ bright supergiants. After this, we only have a B9 $\mathrm{Ia}^{(+)}$object before we reach the A hypergiants (which include the present phase of the LBV W243, Ritchie et al. 2009b). This distribution hints at some sort of break around spectral type B2-3, perhaps suggesting that stars in this mass range finish their H-core burning phase as $\sim$ B2 supergiants.

Though our sample of OB supergiants is far from complete, there have been no systematics in the choice of targets. The level of incompleteness is difficult to assess, as photometry is not available for all the stars in the crowded cluster core area. We estimate that perhaps $\sim 10$ stars which appear bright enough in $I$ to be supergiants have not been observed in the cluster core (most notably W12b, W39a \& W40a), with a similar number in the outskirts (e.g., W53 or W56a). Of course, a much larger population of less luminous OB stars remain to be explored.

\subsection{Mass}

The mass of Wd 1 has been estimated in two different ways. Mengel \& Tacconi-Garman (2008) have used the radial velocity dispersion of the ten stars brightest in the infrared $(\sigma=$ $8.4 \mathrm{~km} \mathrm{~s}^{-1}$ ) to estimate a mass of $\sim 1.3 \times 10^{5} M_{\odot}$. This estimate needs to be confirmed, as it is subject to a large uncertainty. The YHGs show large variations in their radial velocities due to pulsations (Ritchie et al. 2009a; Clark et al. 2010), and some of the stars used present important spectral peculiarities (e.g., W26 is associated with extended nebular emission). Because of this, and the assumption of virial equilibrium, this value should be taken 
as an upper limit. Brandner et al. (2008), on the other hand, have used star counts in the infrared to set a lower limit on the cluster mass $M_{\mathrm{cl}} \gtrsim 5 \times 10^{4} M_{\odot}$. Again, there are important uncertainties coming into this estimation, such as the completeness correction.

In particular, the pre-main-sequence isochrones used by Brandner et al. (2008) indicate a significantly younger $(\tau=$ $3.3 \pm 0.2 \mathrm{Myr})$ and less distant $(d=3.6 \pm 0.2 \mathrm{kpc})$ cluster. This results in very different masses for a given star. Recently, Naylor (2009) has shown that similar discrepancies are observed for a large number of young open clusters and suggested that this effect reflects a real deficiency in the calculation of pre-mainsequence ages.

In any case, the integrated initial mass function, which is independent of the distance and age estimate, appears consistent with Salpeter's down to solar mass (Brandner et al. 2008). The spectroscopic confirmation of a large number of photometric candidate members from $\mathrm{C} 05$ here indicates that the number of stars more massive than the main sequence turn-off is likely to be $\sim 200$. If we accept that the turn-off stars have $M_{*} \gtrsim 25 M_{\odot}$, a Kroupa IMF would then imply a mass $\sim 10^{5} M_{\odot}$, consistent with both determinations.

An important source of uncertainty in both measurements is the role of binarity, which affects both radial velocities and transformation of magnitudes into masses. Different lines of evidence support a very high fraction of binaries with similar mass components amongst cluster members. Most WR stars show indirect indications of binarity: presence of dust in WC stars (Crowther et al. 2006a) and hard X-ray emission in WN stars (Clark et al. 2008). Some of them also show photometric variability and at least one is an eclipsing binary (Bonanos 2007). The pilot study by Ritchie et al. (2009a) shows that the high binary fraction extends to lower masses.

\subsection{Distance and age}

A recent determination of the distance to $\mathrm{Wd} 1$, making use of atomic hydrogen in the direction to the cluster, gives $d=3.9 \pm$ $0.7 \mathrm{kpc}$ (Kothes \& Dougherty 2007), compatible with, though slightly shorter than, estimates based on the stellar population (e.g., Crowther et al. 2006a). If we use the standard reddening law to deredden the magnitudes and assume $D M=13.0$, we find that luminosity class Ia stars typically have $M_{V}$ between -6.4 and $-6.9^{2}$. This is not in bad agreement with existing calibrations (e.g., Humphreys \& McElroy 1984), though the slightly longer distance of Crowther et al. (2006a) $(D M \approx 13.4)$ would give a better agreement. The three mid-B supergiants W57a, W70 and W71 are definitively brighter, with $-8<M_{V}<-7$. W7 and W33 are even brighter, with $M_{V} \approx-8.5$ (which would become almost -9 for $D M=13.4$ ), justifying their tentative classification as hypergiants.

In Fig. 8, we plot an estimate of the intrinsic luminosity for all the objects with good photometry in Table 1 distributed by spectral type and luminosity class. For this, we use $E(V-I)$, under the assumption of a standard reddening law (Rieke \& Lebofsky 1985), to calculate $A_{I}$, and then derive $M_{I}$ for a nominal $D M=13.5$. Due to the uncertainty in $E(B-V)$, we prefer to perform our calculations with the red magnitudes. However, for ease of comparison, we transform to $M_{V}$ simply by adding the intrinsic $(V-I)_{0}$ tabulated for the spectral type. The plot shows, in general, a good correlation between absolute magnitude and

2 Though a few are much fainter. In particular, W55 has -5.6, introducing some doubts about its classification, which is based only on a FLAMES spectrum.

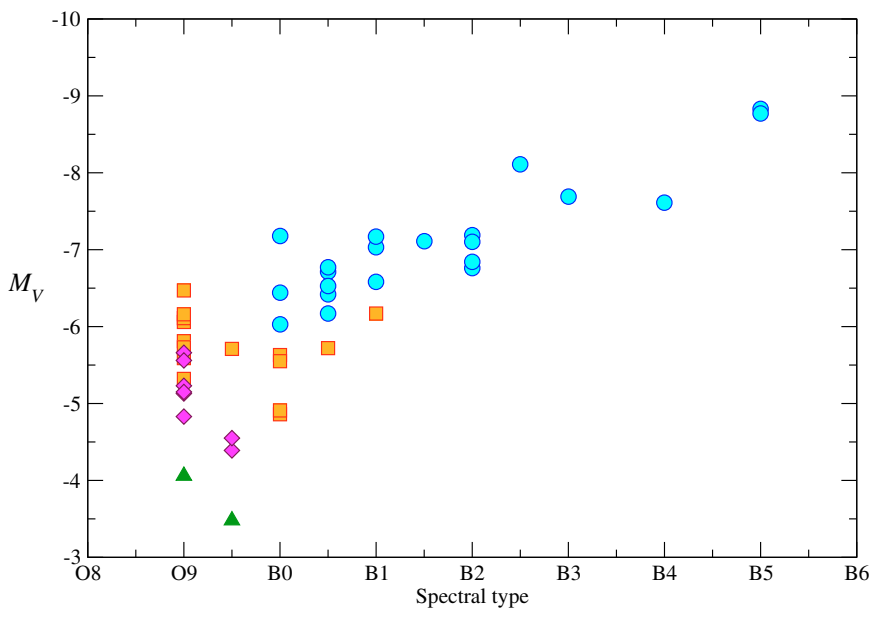

Fig. 8. Observational HR diagram for $\mathrm{Wd} 1$. The absolute magnitudes, $M_{V}$, are estimated from the observed $I$, dereddened using $E(V-I)$ under the assumption of a standard law and corrected with the tabulated $(V-I)_{0}$ for the spectral type from Wegner (1994). Circles represent objects classified as Ia supergiants; squares are Iab classifications, diamonds are Ib objects and triangles are stars with lower luminosity.

luminosity class, giving strong support to our classifications. The obvious exception are the objects classified as O9 SGs. These objects cover a broad span (>2 mag) in $M_{V}$, present an overlap between O9 Iab and O9 Ib objects (though objects classified O9 Iab are in most cases brighter than objects classified O9 Ib) and have $M_{V}$ 's rather higher than expected (the O9 Iab objects are as bright as the B0 Ia stars).

This discrepancy may arise from the difficulty in separating the effects of luminosity and fast rotation in O-type stars at this moderately low resolution. Some of the objects classified as O9 Iab may be fast-rotating O9 Ia stars. Though plausible, this interpretation presents two difficulties: first, all O9 Ia stars present should be fast rotators (perhaps not a serious objection when we are talking of 3-4 stars); second, it would mean that we have a very large range of luminosity classes at spectral type O9 (from O9 III to O9 Ia), as opposed to other spectral subtypes. Alternatively, as discussed above, we can think that some of the objects classified as O9 Iab are really later-type ( B0) stars with enhanced C III $8500 \AA$, but this does not necessarily imply brighter $M_{V}$. Perhaps the simplest interpretation is that the objects we have classified as O9 Iab are really O9.5 Ia. As seen in Fig. 8, this small change in their classification would make their luminosities compatible with all the other members ${ }^{3}$.

With this caveat, we may try to constrain the age of the cluster from the observed population. For this, we are forced to rely on the predictions of evolutionary models. As discussed above, the tracks followed by high-mass stars in the theoretical HR diagram are subject to large uncertainties. As the exact mechanism for heavy mass loss is unknown, mass loss rates are normally introduced by hand, following some recipe. Differences in mass loss rates, the treatment of rotation and the degree of convective overshooting assumed lead to rather different evolutionary tracks (e.g., Salasnich et al. 1999; Meynet \& Maeder 2000; Eldridge et al. 2008). Furthermore, the inclusion of binarity can substantially alter the evolution of massive stars (e.g., Vanbeveren et al. 2007; Eldridge et al. 2008).

\footnotetext{
3 As discussed in Sect. 3, many of the O9 Iab classifications are less reliable than the rest, due to lower resolution or smaller spectral range observed.
} 
As discussed by Crowther et al. (2006a), single evolutionary models have trouble reproducing the observed population of Wd 1. Geneva isochrones without rotation (Meynet \& Maeder 2000) predict that RSGs will be formed only for ages $\gtrsim 6 \mathrm{Myr}$, while even higher ages are required to produce RSGs with the high-rotation isochrones. On the other hand, the WR populations decline rapidly after 5 Myr. The simultaneous presence of large numbers of cool super/hypergiants and WR stars is reproduced better by models including binaries, which also provide a better description of the ratio between WC and WN stars. Comparison of the number ratio between WR stars and cool hypergiants with isochrones from Eldridge et al. (2008) led Crowther et al. (2006a) to propose an age of 4.5 or $5.0 \mathrm{Myr}$, with the progenitors of the WR stars having initial masses in the 40-55 $M_{\odot}$ range.

Such age seems consistent with the observed distribution of blue supergiants. We observe O9-B0 supergiants with different luminosity subclasses, while at later spectral types we only see Ia supergiants. At $\sim 4.5 \mathrm{Myr}$, these Ia supergiants should be descended from stars with initial masses $M_{\text {ini }} \sim 35 M_{\odot}$. This is in good agreement with placement on theoretical tracks of B Ia supergiants. For instance, Crowther et al. (2006b) find that a sample of B0-3 Ia Galactic supergiants lie between the tracks for 25 and $40 M_{\odot}$.

As single star isochrones have been frequently used to date open clusters, here we will compare the properties of OB supergiants to Geneva isochrones. As the stars are still relatively unevolved, the differences with respect to other single star tracks are minor, allowing easy comparison to the ages of other open clusters.

In Fig. 9, we plot the same estimates of $M_{V}$ derived above against $T_{\text {eff }}$ for all the Ia and Iab supergiants. The effective temperatures used are an interpolation between the observational scale of Martins et al. (2005) for late O-type stars and the temperature scale of Crowther et al. (2006b) for B-type supergiants. We also plot several isochrones from the models of Meynet $\&$ Maeder (2000). Taking into account that the $M_{V}$ determination is subject to important uncertainties (for example, simply the rounding up of the second decimal in the determination of the ratio between $A_{I}$ and the colours amounts to a difference $>0.1 \mathrm{mag}$ ) and the approximate character of the $T_{\text {eff }}$ scale, the general agreement is rather good. We note the following points:

- the $\log t=6.7$ (5 Myr) isochrone without rotations would provide a good fit for a distance approaching $6 \mathrm{kpc}$;

- the $\log t=6.8$ (6.3 Myr) isochrones (with and without rotation) provide a very good fit to the position of the B supergiants for distances $\sim 4.5-5 \mathrm{kpc}$. These isochrones would, however, fail to reproduce the position of the $\mathrm{O}$ supergiants and almost certainly fail to reproduce the turn-off of the main sequence, unless all the objects we have classified as O-type supergiants are blue stragglers;

- the location of the mid-B supergiants is compatible with their being evolving away from the main sequence;

- the distance from Kothes \& Dougherty (2007) ( $D M=13.0)$ implies an age $>7 \mathrm{Myr}$ for the cluster, which seems incompatible with the population of WR stars. Their error bars, however, reach within the area favoured by our data.

If we accept that a significant fraction of our stars are binaries containing two massive stars, they would be intrinsically brighter, allowing a longer distance (by $\sim 0.5 \mathrm{mag}$ ) for a given age.

Considering the uncertainties involved here, a definite answer will have to wait for accurate spectral classification of objects near the turn-off of the main sequence, which can be

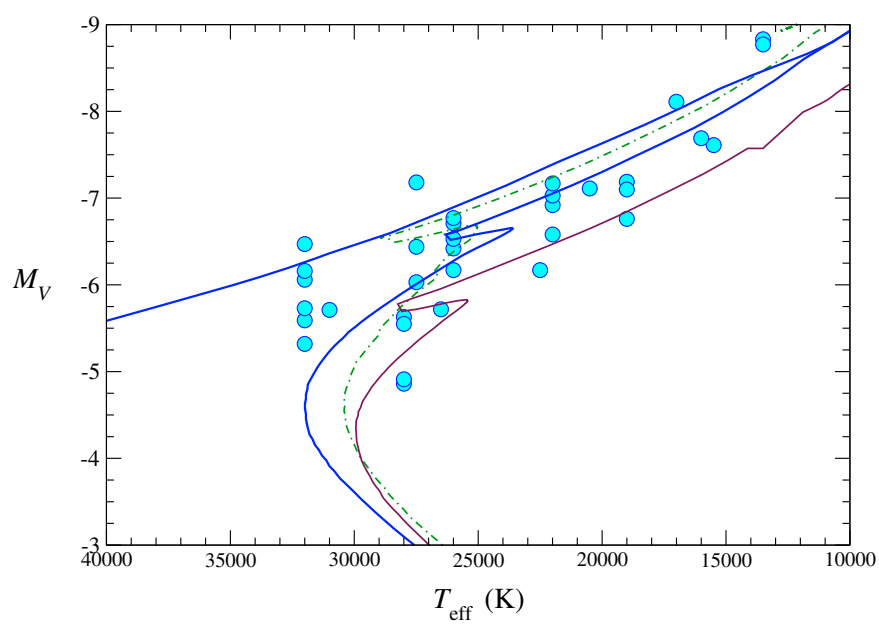

Fig. 9. Semi-empirical HR diagram for bright OB supergiants in Wd 1. The solid lines represent the Geneva isochrones without rotation for $\log t=6.7$ (5 Myr; top, blue) and $\log t=6.8$ (6.3 Myr; bottom, brown). The dash-dotted line is the $\log t=6.8$ isochrone for high initial rotational velocity.

achieved with high quality infrared spectra. This will allow the anchoring of the infrared photometric sequence, and a much more accurate isochrone fit. The richness of the OB population in Wd 1 may then allow the creation of a more accurate temperature scale based on fits of tailored synthetic spectra to high quality spectra of our sample. Meanwhile, our data strongly favour $d \gtrsim 5 \mathrm{kpc}$ and $\tau \gtrsim 5 \mathrm{Myr}$ for $\mathrm{Wd} 1$, and seem to definitely rule out the values of Brandner et al. (2008), based on pre-mainsequence tracks. As this paper contains a careful analysis of the data, we suspect that this result reflects inaccuracies in the preMS isochrones, as found by Naylor (2009) for other young open clusters.

\subsection{The yellow hypergiants}

Wd 1 contains six luminous hypergiants with spectral types between $\mathrm{A}_{5} \mathrm{Ia}^{+}$and F8 Ia ${ }^{+}$(Clark et al. 2010). They represent a sizable fraction of the known Galactic YHGs. The evolutionary status of YHGs is unclear under current models. In several schemes (e.g., Smith et al. 2004), they are assumed to be stars that have already been RSGs and are coming back towards the blue in their way to become Wolf-Rayet stars. With the current dataset, the evolutionary status of the YHGs in Wd 1 is difficult to assess. A simple estimation of their luminosities using the same procedure utilised for the OB supergiants suggests that their bolometric luminosities are not very different from those of the B hypergiants (and, in the case of W8a, substantially lower) ${ }^{4}$.

We have to be careful with this estimation, though. Spectroscopic monitoring of the YHG W265 has shown it to present strong pulsational variability, which leads to changes in spectral type between $\mathrm{F} 1 \mathrm{Ia}^{+}$and $\mathrm{F}_{5} \mathrm{Ia}^{+}$, with a period of $\sim 100 \mathrm{~d}$ (Clark et al. 2010). There is a high likelihood that the other YHGs experiment similar variations. Such variability in spectral type should be reflected in significant changes in the intrinsic colours and bolometric corrections. Our photometric and spectroscopic data are not simultaneous, and so the two datasets may correspond to different spectral types. Moreover, and perhaps more importantly, strong pulsations may lead to heavy mass loss,

\footnotetext{
4 Note that we lack photometry for such interesting objects as W42a $\left(\mathrm{B} 9 \mathrm{Ia}^{+}\right), \mathrm{W} 32\left(\mathrm{~F} 5 \mathrm{Ia}^{+}\right)$and W243 (LBV/A3 $\left.\mathrm{Ia}^{+}\right)$.
} 


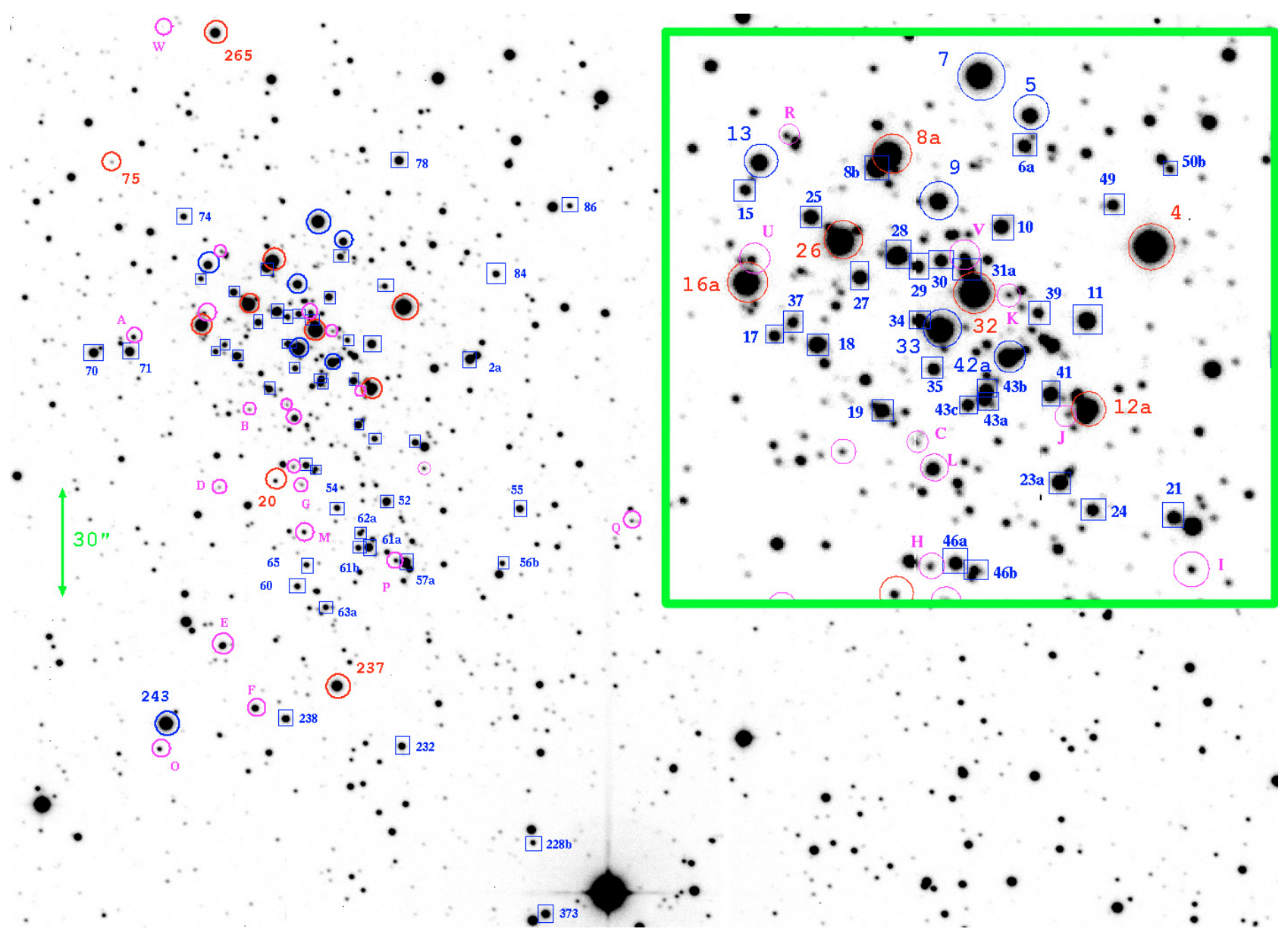

Fig. 10. $R$-band finder $\left(\sim 6^{\prime} \times 4^{\prime}\right)$ for all the stars classified as OB supergiants in this paper. Other stars in more advanced evolutionary stages are also shown. The moderately evolved OB supergiants are shown as rectangles. The more evolved stars are shown as circles: blue circles are blue hypergiants and LBVs, red circles are A/F hypergiants and M supergiants, while magenta circles are WR stars. The inset $\left(\sim 70^{\prime \prime} \times 70^{\prime \prime}\right)$ shows the finder of the crowded cluster core. Many stars in this area (and also in the halo) have not been observed. Note that WR stars N, X and T are outside the area covered by this finder.

and the assumption of typical colours for A-F supergiants may not be appropriate if the stars suffer from intrinsic absorption.

Therefore a detailed spectroscopic analysis, including model fits for abundance determination, seems instrumental in deriving the evolutionary status of the YHGs. So far, such an analysis has only been conducted for the LBV/A3 $\mathrm{Ia}^{+} \mathrm{W} 243$, which shows very clear signs of heavy element processing in its atmosphere. As noted by Crowther et al. (2006a), single star evolutionary models do not reproduce the simultaneous presence of luminous cool supergiants and WR stars in Wd 1. Models including binary evolution fare better in this respect but, as pointed out by Clark et al. (2010), no current model seems able to accommodate the large population of cool supergiants at the estimated age. Understanding the evolutionary relations between the evolved stars in Wd 1 will therefore provide very strong constraints on evolutionary models.

\subsection{Spatial distribution}

Based on star counts in the infrared, Brandner et al. (2008) found the cluster to be clearly elongated. The diffuse X-ray emission from the cluster shows a similar elongation (Muno et al. 2006), with the major axis at Position Angle $13^{\circ} \pm 3^{\circ}$.
In Fig. 10, we compare the distribution of the OB SG to that of the more evolved stars. For this latter set (comprising A/F hypergiants, $M$ supergiants and WR stars), we can be certain of having a complete sample. The distributions of the two sets are not significantly different, revealing a very compact core, which contains more than half the objects of interest. This core measures only $\sim 60^{\prime \prime} \times 45^{\prime \prime}$, with the centre approximately defined by the positions of W27, W29 and W30, and is elongated along the approximate NE-SW line. In addition, there is a secondary clump to the South, with a number of objects likely defining a more symmetrical halo. We note, however, that the division in two clumps is not obvious in either the diffuse X-ray emission or the infrared star counts. Indeed, Brandner et al. (2008) place the centre of the cluster in a position very close to WR L, in the area of lower density of supergiants. This may suggest that the division in two clumps is not real, but due to a random decrease in the number of optically bright stars. In such case, the shape of the cluster, as defined by its supergiant (and WR) populations would resemble more closely an arc than an elongated structure.

Some objects, like W228b and W373, are located more than $150^{\prime \prime}$ away from the putative core centre, hinting at the possibility that Wd 1 is surrounded by a very extended halo. Though this possibility has not been explored yet, it is given strong support by the presence of 3 WR stars likely associated with the 
cluster at much higher distances (WR stars N, X and T in the notation of Negueruela \& Clark 2005; Crowther et al. 2006a).

At this level of analysis, we find no strong indication of any difference in the relative distributions of evolved and unevolved stars, which could point to an age difference between regions inside Wd 1 . The visually brightest stars seem to be concentrated in the small core defined above, but, given that neither the less evolved supergiants (their progenitors) or the WR stars (their descendants) share this concentration, this may be a random effect. In view of this, we conclude that the data so far strongly point to a single, homogeneous age (though some minimal spread, $\sim 1 \mathrm{Myr}$, is allowed) for the whole cluster.

\section{Conclusions}

Using new classification criteria, developed in Appendix A, we provide spectral types for almost $60 \mathrm{OB}$ supergiants in $\mathrm{Wd} 1$. Only a small fraction of them had previous accurate classifications.

The stars leaving the cluster main sequence form a well populated clump at spectral types O9-B0, displaying a range of luminosities, which likely reflect a difference in initial rotational velocities and, perhaps, formation over a timespan. Sixteen luminous supergiants, with spectral types in the range B1-B4 range span the temperature range between this clump and the three blue hypergiants that bridge the gap with the A/F hypergiants.

After a careful analysis of colour excesses, we find no strong reason to suspect that the extinction law towards $\mathrm{Wd} 1$ deviates strongly from the standard, and we attribute the slightly divergent $E(B-V)$ values to the difficulty in obtaining precise $B$ photometry. The measured values of $E(V-I)$ and $E(V-R)$ show that the extinction varies strongly across the face of the cluster, with average values implying $A_{\mathrm{V}}=10.8$. This value is fully consistent with the $A_{K_{\mathrm{S}}}=1.13 \pm 0.03$ derived by Brandner et al. (2008) and a standard law. The value $A_{K_{\mathrm{S}}}=0.96$ found by Crowther et al. (2006a), though subject to much higher uncertainty, is also compatible.

The dereddened magnitudes of the OB supergiants support a distance to Wd 1 similar to that found by Crowther et al. (2006a) from the infrared magnitudes of WR stars, namely $D M=13.5$ $(d=5.0 \mathrm{kpc})$. With this distance modulus, the intrinsic magnitudes of OB supergiants correspond closely to their spectral types according to typical calibrations. The shorter $D M=13.0$ $(d=4.2 \mathrm{kpc})$ found by Kothes \& Dougherty (2007) is disfavoured (though their error bars reach $d=4.9 \mathrm{kpc}$ ). The $D M=12.75(d=3.5 \mathrm{kpc})$ found by Brandner et al. (2008) is strongly disfavoured, as it would mean that all our Ia supergiants would have intrinsic magnitudes typical of Iab supergiants, implying masses $\$ 25 M_{\odot}$, very difficult to reconcile with their age derivation of 3.6 Myr. We suspect this to be due to the systematically younger ages that pre-MS isochrones indicate (cf. Naylor 2009).

The picture drawn from the distribution in spectral types seems compatible with a single burst of star formation and the predictions of evolutionary models until the stars start to move quickly towards lower temperatures. Further studies, including the use of the population of unevolved stars to nail down the cluster age and detailed analysis of the evolved population (which will provide accurate stellar parameters and element abundances), will undoubtedly result in very firm constraints on the evolutionary tracks followed by massive stars after they leave the main sequence. The high binary fraction found amongst massive stars in Wd 1 (Clark et al. 2008; Ritchie et al. 2009a) will likely allow a better understanding of the role that binarity plays in this evolution. Furthermore, characterisation of binary properties (frequency, mass ratio, period distribution, etc.) in the homogeneous massive star population of $\mathrm{Wd} 1$ can provide strong constraints on the formation mechanism of massive stars in such dense environments ${ }^{5}$, as the different formation mechanisms proposed will predict different outcomes (Zinnecker \& Yorke 2007, for a review). Long-term spectroscopic monitoring of binaries in the cluster is underway (Ritchie et al. 2009a), and will deliver some of the observational characteristics.

Acknowledgements. We thank Lucy Hadfield for help with the 2004 observations and Dr. Amparo Marco for participating in other runs. We also thank Antonio Floría for help with the artwork in the finder. Finally, we thank the referee, Michaël De Becker, for helpful comments. This research has been funded by grants AYA2008-06166-C03-03 and Consolider-GTC CSD-2006-00070 from the Spanish Ministerio de Ciencia e Innovación (MICINN). J.S.C. acknowledges the support of an RCUK fellowship. This research has made use of the Simbad database, operated at CDS, Strasbourg (France).

\section{Appendix A: Spectral classification of $\mathrm{OB}$ supergiants in the red}

\section{A.1. Historical introduction}

OB stars have spectra characterised by strong Balmer and He lines. Metallic lines are few and weak, except in the range O9-B2, especially in main sequence stars. The blue range concentrates most of the salient spectral features and spectral classification has been developed using features in the $\lambda \lambda$ 3900-4700 range (e.g., Walborn 1971; Walborn \& Fitzpatrick 1990). The red part of the spectrum contains fewer features and has generally been ignored, except for $\mathrm{H} \alpha$, considered a good tracer of mass loss. An overview of features in the $\lambda \lambda$ 5400-6600 $\AA$ range is presented by Walborn (1980), together with a spectral atlas based on photographic spectrograms. High-quality digital spectrograms in this range of a few selected objects are presented in Walborn \& Howarth (2000).

Further to the red, atmospheric features become a complication. There is, however, an important window, free from atmospheric features, in the $\lambda \lambda 8300-8900$ range. Andrillat et al. (1995) present spectra of early-type stars in the $\lambda \lambda$ 8375-8770 range, concluding that supergiants can be distinguished from main sequence stars from the shape of the Paschen lines. According to these authors, stars earlier than B3 cannot be classified in this region. Higher resolution spectra were presented by Munari \& Tomasella (1999). Their spectral atlas covers the $\lambda \lambda$ 8480-8750 range at $R=20000$, but little attention is paid to early type stars. Munari \& Tomasella (1999) find some degeneracy between luminosity class and rotational speed and conclude that O-type stars cannot be classified at all with this limited range.

Caron et al. (2003) studied quantitative classification criteria, using intermediate-resolution spectra in the $\lambda \lambda$ 8400-8900 range for stars with spectral types between O9 and B5. They found that the lines are always broader in dwarfs than in supergiants. In addition, the equivalent widths of most lines increase with advancing spectral type. Based on these findings, they developed a method for approximate classification based on line ratios. This approach was followed in $\mathrm{C} 05$, where the grid was

\footnotetext{
5 Note, however, that at an age $\sim 5$ Myr, Wd 1 must have already undergone dynamical evolution. Indeed, Brandner et al. (2008) find some evidence for mass segregation, which could partially erase the signature of the formation mechanism.
} 
extended by using synthetic spectra and the validity of the classifications was checked against spectra of stars of known spectral types (see Appendix A in C05).

Quantitative analysis of features in the red region have hardly been reported. The only exceptions are C II $6578 \AA$, Si II $6347 \AA$ and Ne I $6402 \AA$, included by Lennon et al. (1993) in their analysis of features seen in B-type supergiants. In addition, Davies et al. (2005) present an atlas of luminous supergiants in this range.

\section{A.2. Data used}

In an attempt to improve our knowledge of the behaviour of features in the red and far red regions, we have collected spectra of $\mathrm{OB}$ stars in this range from a number of sources. The STELIB library (Le Borgne et al. 2003) contains stellar spectra at $R \sim 2000$. The data cover the whole optical range, but the number of OB supergiants observed is small. The library by Cenarro et al. (2001) covers the $\lambda \lambda 8350-8900$ range at better spectral resolution, but, being conceived to study the Ca II triplet, contains few early-type stars. The more recent MILES library (Sánchez-Blázquez et al. 2006), which includes more early-type stars, only extends until $7500 \AA$.

The main sources of data have been the Indo-US library (Valdes et al. 2004), which includes spectra in the range $\lambda \lambda 3460-9460$ at intermediate resolution and the UVES Paranal Observatory Project (ESO DDT Program ID 266.D-5655) spectra (Bagnulo et al. 2003). The UVES POP spectra have excellent resolution $R \approx 80000$ and SNR. Unfortunately, as the data were taken as part of a poor weather backup programme, the red sections are generally affected by very strong atmospheric features. Moreover, the spectra present a gap in the $\lambda \lambda 8540-8660$ interval, due to the configurations used.

In all, the spectra represent a rather complete, if heterogeneous, sample of B-type luminous stars. The O subtypes are more sparsely sampled.

\section{A.3. Results}

In spite of the scarcity of spectral features in the red spectra of OB stars, several criteria can be used for spectral classification. Unfortunately, most of these criteria are sensitive to both spectral type and luminosity, but by considering the whole spectrum in the $\lambda \lambda 6000-9000$ range and combining several criteria, we can narrow the spectral range for a given supergiant spectrum generally down to half a spectral subtype. The classification of dwarf stars is much less accurate. In what follows, we will concentrate on the features used to classify the supergiants seen in $\mathrm{Wd} 1$.

\section{A.3.1. Classification in the $I$-band}

Criteria for the classification of O9-B5 stars in this range have been laid down by Caron et al. (2003). They base their classification in the relative strengths of Paschen lines. The best temperature criteria seem to be the $E W(\mathrm{~Pa} 16+\mathrm{C}$ III $) / E W(\mathrm{~Pa} 11)$ or $E W(\mathrm{~Pa} 15) / E W(\mathrm{~Pa} 11)$ ratios, even though $\mathrm{He} \mathrm{I} 8850 \AA$ is blended with $\mathrm{Pa} 11$ at moderate resolutions. The ratio $F W H M(\mathrm{~Pa} 13) / F W H M(\mathrm{He}$ I $8779 \AA)$ is a good luminosity criterion at the resolution they use. Unfortunately, in the spectra of stars in Wd 1, suffering from heavy extinction, there seems to be a complex system of diffuse interstellar bands. The $8620 \AA$ band is sharp and narrow. Munari et al. (2008) has reported an excellent correlation between its strength and reddening up to rather high reddenings, at least $E(B-V) \sim 2$. However, at the much higher reddening of $\mathrm{Wd} 1$, this feature is contaminated by neighbouring ones. There is a broad, relatively shallow feature, perhaps similar in shape to the $4430 \AA$ DIB, centred around $8648 \AA$. This feature is blended with a He I $8653 \AA$ line, weakly seen in many spectra of B-type stars in Caron et al. (2003).

In addition, there appears to be a global depression of the continuum in the whole range $8540-8660 \AA$. The actual nature of this depression is difficult to decide, but it has a strong effect on continuum normalisation. Treating it as a single feature produces an awkward shape for the continuum and results in a wrong sequence of strengths for the Paschen lines. In view of this, for the purposes of this paper, we have normalised the spectrum as if the continuum around $8610 \AA$ were not affected by this broad depression (effectively assuming that there are two such depressions, one on each side of $8610 \AA$ ) and we have normalised the spectra taking reference points in the continuum at 8520,8610 and $8690 \AA$. This procedure results in a decrease of the strength of the Paschen lines as we move up the sequence and it is hence taken to be approximately correct. However, it is only an approximation and we assume that the equivalent widths of $\mathrm{Pa} 13,14$ and 15 cannot be measured with accuracy. Because of this, we are forced to try to define a morphological classification scheme.

Several He I lines are seen in the $I$-band spectra of B-type stars. Though never near as prominent as the Paschen lines, they are much better defined in supergiants, showing maximum strength at B2 ${ }^{6}$. In dwarf stars, He I lines are always weak and only seen in the O8-B2 range (perhaps visible in high-SNR spectra of B3 V slow rotators). In giants, they are visible up to B4-5, depending on $v_{\text {rot }}$. In supergiants, they are seen down to B6 or even later if the SNR is very good.

The O I $8446 \AA$ line has a strong dependence on both spectral type and luminosity. As shown in Fig. A.1, at a given spectral type, the ratio with the neighbouring Pa 18 (8437 $\AA$ ) decreases slowly with increasing luminosity. Figure A.2, on the other hand, shows how the same ratio increases with spectral type at a given luminosity. To make things worse, the shape of the line depends strongly on the rotational velocity. This can be easily seen by comparing the spectrum of HD $120315\left(\mathrm{~B} 3 \mathrm{~V}, v_{\text {rot }}=150 \mathrm{~km} \mathrm{~s}^{-1}\right)$ and HD 160762 (B3 IV and a very slow rotator; see Abt et al. 2002). In any case, O I $8446 \AA$ is first seen at B2 in dwarf stars and starts to appear on the wing of Pa 18 at B1.5 in supergiants.

An important diagnostic for B3 is the appearance of $\mathrm{N} I$ lines. They are weak at this spectral type, but quickly become stronger at later types, becoming prominent in supergiants after B5. These lines are not seen in dwarf stars. They are not seen in most spectra of luminosity class III objects, even the high-resolution UVES spectra. They are clearly visible in the few luminosity class II spectra available (including the B3 II star in Fig. A.1), and so it is reasonable to expect that they may appear weakly in some giants with low $v_{\text {rot }}$. In fact, they are weakly visible in the spectrum from Andrillat et al. (1995) of HD 186568 (B8 III), which is a very slow rotator $\left(v_{\text {rot }}=15 \mathrm{~km} \mathrm{~s}^{-1}\right.$; Abt et al. 2002).

As discussed in C05, O-type stars can be easily identified by the presence of He II $8238 \AA$ A. Unfortunately, this line lies in the middle of a strong atmospheric band. When telluric features are accurately removed, He II $8238 \AA$ can be readily identified (Fig. A.3). Then it is visible up to at least O9.5, though it may be difficult to locate in fast rotating dwarfs. When the telluric

\footnotetext{
${ }^{6}$ The He I 8582.7 \& $8776.7 \AA$ lines were first described by van Helden (1972), who discusses their sensitivity to luminosity.
} 


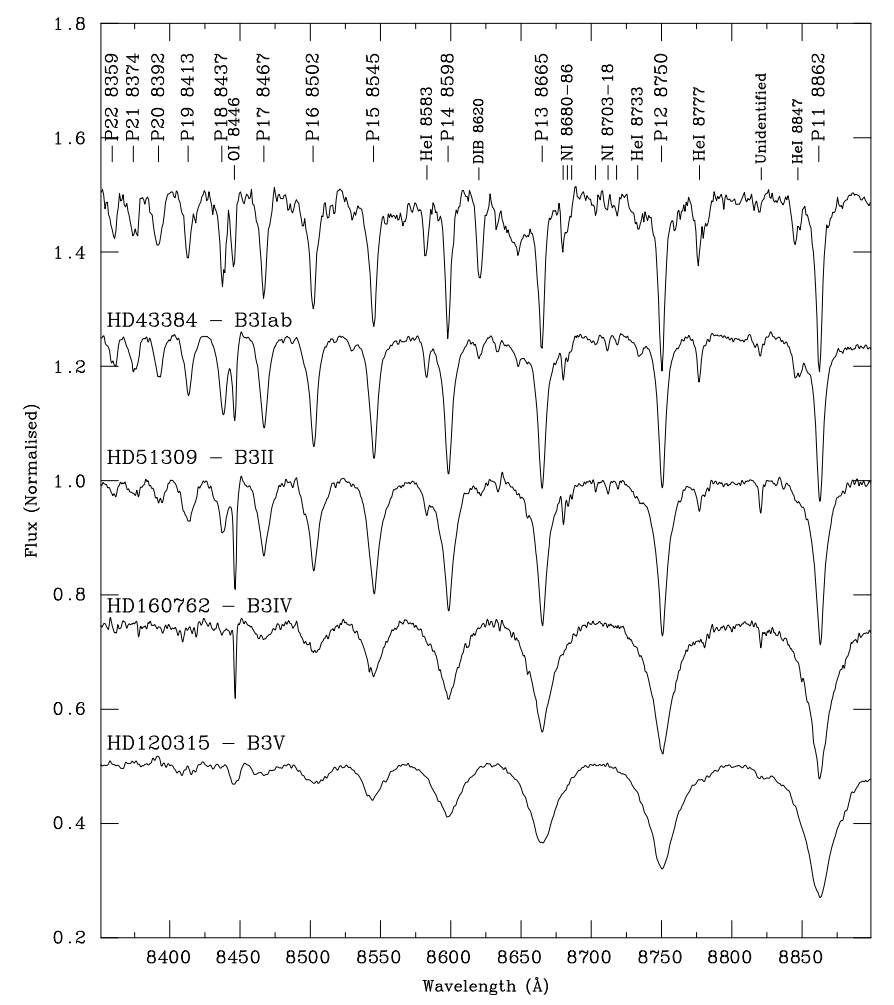

Fig. A.1. Luminosity sequence at B3. The unlabelled spectrum at the top is W70 (B3 Ia). All the other spectra have been taken from the IndoUS library. Note the strengthening and sharpening of He I and $\mathrm{N}$ I lines with increasing luminosity and the changing Pa 18/O I $8446 \AA$ ratio. The spectrum of W70 is affected by the strong interstellar features mentioned in the text.

features cannot be removed, as in the UVES POP spectra, it is almost impossible to see, though it may be guessed in the spectra of early and mid O-type stars. Based on the few spectra available, this line may show some dependence on spectral type, being weaker in O8-9 stars. No strong dependence on luminosity class can be identified.

The second important marker of O-type stars is C III $8500 \AA$. This line almost coincides with $\mathrm{Pa} 16(8502 \AA)$ in wavelength. In O6-7 dwarfs, where $\mathrm{Pa} 16$ is hardly seen, it appears as a weak narrow line (Fig. A.3). In O8-B0 stars it can be noticed as a important strengthening of $\mathrm{Pa} 16$ in comparison with $\mathrm{Pa} 15$ and $\mathrm{Pa}$ 17. This strengthening stops being noticeable around B0.5, in line with the behaviour of C III lines in the blue. As other $\mathrm{C}$ lines, this might be an unreliable tracer in stars with $\mathrm{CNO}$ anomalies, though we lack appropriate spectra to test this issue.

\section{A.3.2. Classification in the red region}

Spectral features in the red region are scarce in OB stars. Many dwarfs show only $\mathrm{H} \alpha$ and the He I $6678 \& 7065 \AA$ lines. Supergiants present a few other interesting features, notably in B-types.

There are two He II lines present in O-type spectra (a third one, He II $6560 \AA$, cannot be separated from $\mathrm{H} \alpha$ ). Unfortunately, He II $6527 \AA$ coincides with a strong DIB and cannot be used for classification. He II $6683 \AA$ starts to be seen as a weak inflection on the wing of He I $6678 \AA$ in high-SNR spectra of O9 stars, and it becomes stronger at earlier spectral types. However, a clear dependence on spectral type cannot be drawn from the few spectra

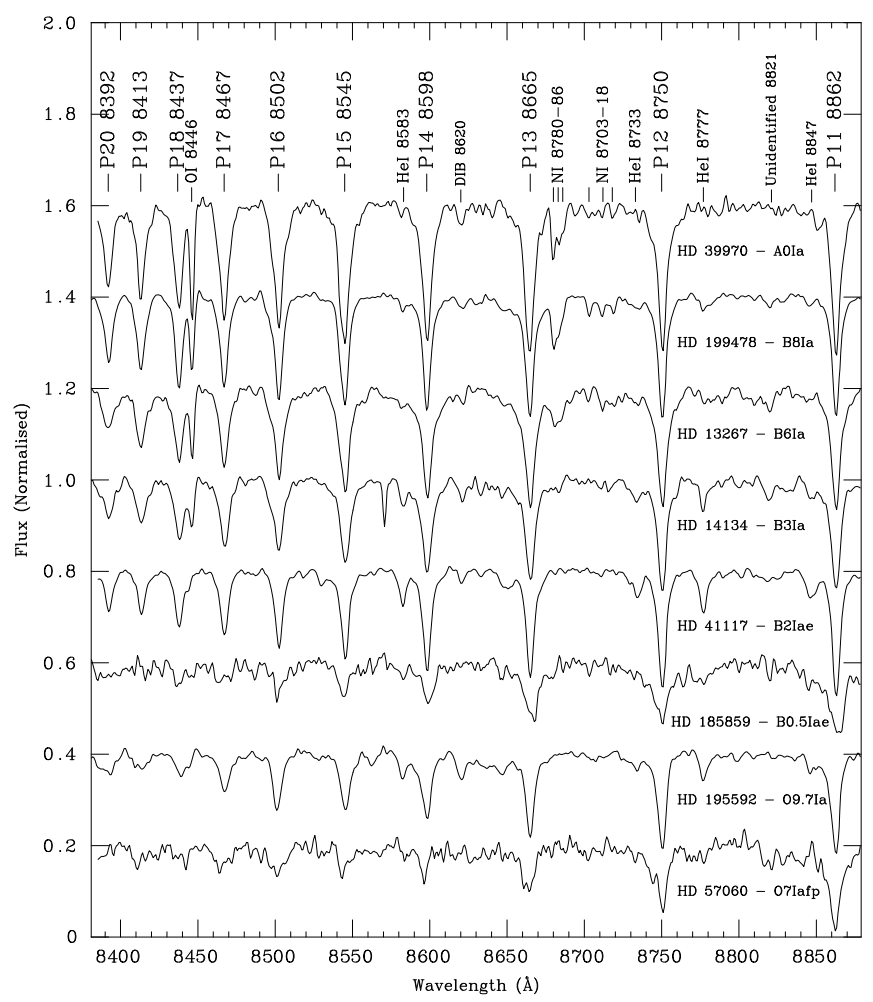

Fig. A.2. Spectral sequence of high-luminosity supergiants. Spectra are taken from Cenarro et al. (2001) and have only moderate resolution and occasionally low SNR. Note the increase of N I lines with advancing spectral type and the sharp maximum of the He I around B2. Note also that the lines marked above the top spectrum are those seen in B-type spectra and do not match those on the A0 Ia spectrum.

available (Fig. A.4). The ratio He I $6678 \AA$ A/He II $6683 \AA$ cannot be used to determine spectral type. Indeed, from the four stars with spectral type O6-6.5, it would seem to depend more strongly on luminosity.

A clear marker of high luminosity in O-type stars is the presence of the SiIV 6668, $6701 \AA$ lines in emission. These are "wind" or selective emission lines, due to non-LTE effects in an extended atmosphere. They are seen in luminous supergiants earlier than O9. We lack information on their behaviour in early O-types. The Si IV lines in the blue part of the spectrum go into emission at much earlier spectral types (Walborn et al. 2002).

Moving to later spectral types, we find another interesting "wind" feature, the C II 7231, $7236 \AA$ doublet. Unfortunately, this feature lies in the middle of an atmospheric band, but, being in emission, is easily identifiable. This feature is visible between 09.7 and B2. Its strength is clearly correlated to that of $\mathrm{H} \alpha$ emission, though there may be some dependence on spectral type, as well, with stronger features being seen in the B0.5-1 range. It is restricted to stars with heavy mass loss, and so generally only appears in luminosity class Ia supergiants.

The behaviour of $\mathrm{H} \alpha$ is similar. This line is a good tracer of mass loss (Leitherer 1988). It tends to be in emission in luminosity class Ia supergiants (as shown in Fig. A.5) and appears normally in absorption in luminosity class Ib stars. Luminosity class Iab supergiants occupy a middle ground, with $\mathrm{H} \alpha$ weakly in absorption or neutralised on many occasions, but in emission or strong absorption in other cases. There are many exceptions to this general rule (see more examples in Davies et al. 2005, Appendix A). In many cases, variable $\mathrm{H} \alpha$ emission is taken as evidence for binarity (e.g., Thaller 1997), but monitoring of a 


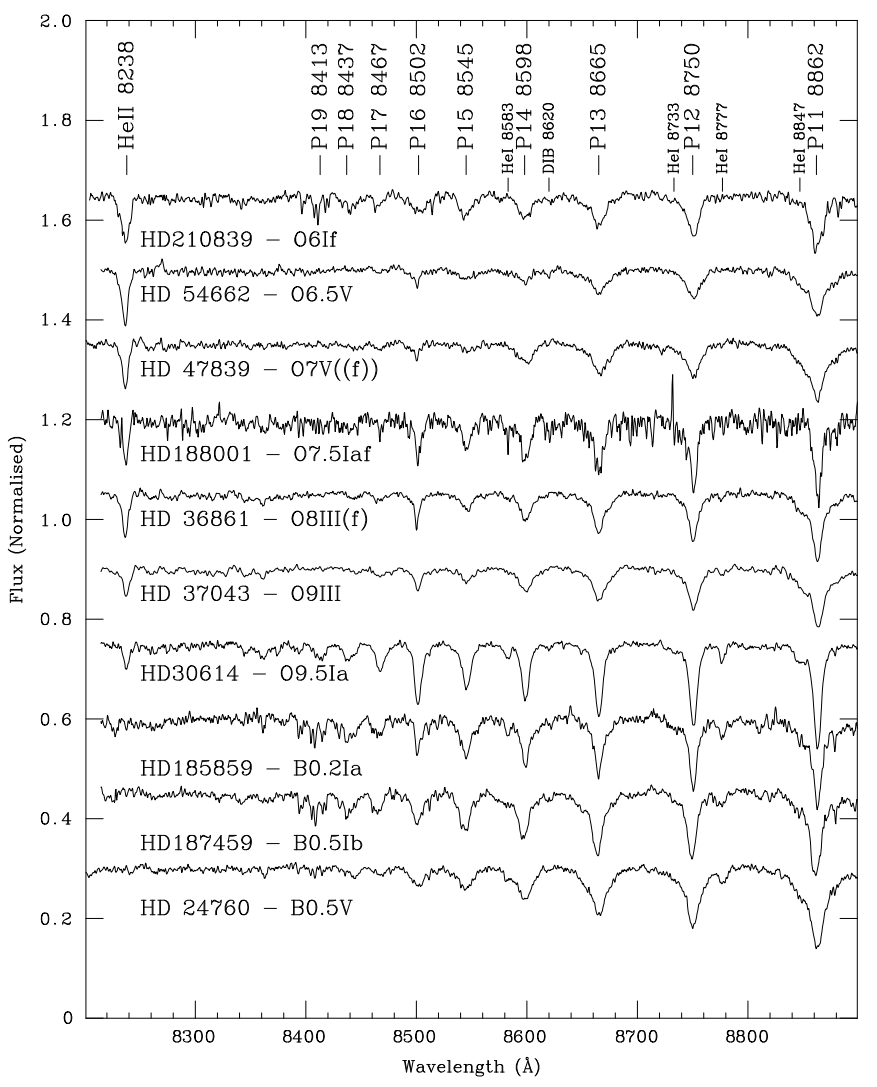

Fig. A.3. I-band spectra of O- and early B-type stars. These spectra from the Indo-US library have had the telluric features removed and allow the study of the behaviour of He II $8238 \AA$. Note how this line clearly identifies O-type stars. Note also the much higher number of Paschen lines seen in supergiants at a given spectral type. Finally, the C III $8500 \AA$ line is an excellent indicator of late O-type stars.

few luminous OB supergiants has shown that important changes can happen on very short timescales (Morel et al. 2004). This behaviour has also been observed amongst the brightest OB supergiants in Wd 1 (Clark et al. 2010).

The shape of $\mathrm{H} \alpha$ in emission has some dependence on spectral type. In B-type supergiants, it appears as a P-Cygni profile, sometimes superimposed on a broader plinth. The absorption trough becomes weaker around B1-2 and tends to disappear completely. B0-0.5 supergiants generally show a strong single peak. O-type supergiants tend to have more complex features, with more than one peak. This is complicated by the presence of the He II $6560 \AA$ line in absorption.

Emission is weaker for late-B supergiants, which generally have weak P-Cygni profiles, but there are also many exceptions. For example, the B8 Ia supergiant HD 199478 can show a strong $\mathrm{H} \alpha$ single peak emission, though this line is variable (Markova \& Valchev 2000). The most luminous hypergiants may also display strong symmetric peaks. For example HD $32034\left(\mathrm{~B} 8-9 \mathrm{Ia}^{+}\right)$or HD 269781 (A2 Iae) in the catalogue of Le Borgne et al. (2003) do, but HD $33579\left(\mathrm{~A} 3 \mathrm{Ia}^{+}\right)$and HD $223385\left(\mathrm{~A}^{2} \mathrm{Ia}^{+}\right)$display a weak absorption trough superimposed on the strong emission peak (note that the extremely high luminosity of all these stars, except HD 223385, can be confirmed, as they are members of the LMC).

The O I $7774 \AA$ triplet is a very good luminosity indicator. Calibrations of its strength against luminosity exist for A-G stars (e.g., Arellano Ferro et al. 2003), but its behaviour has not been tabulated in B-type stars. The lines are barely visible

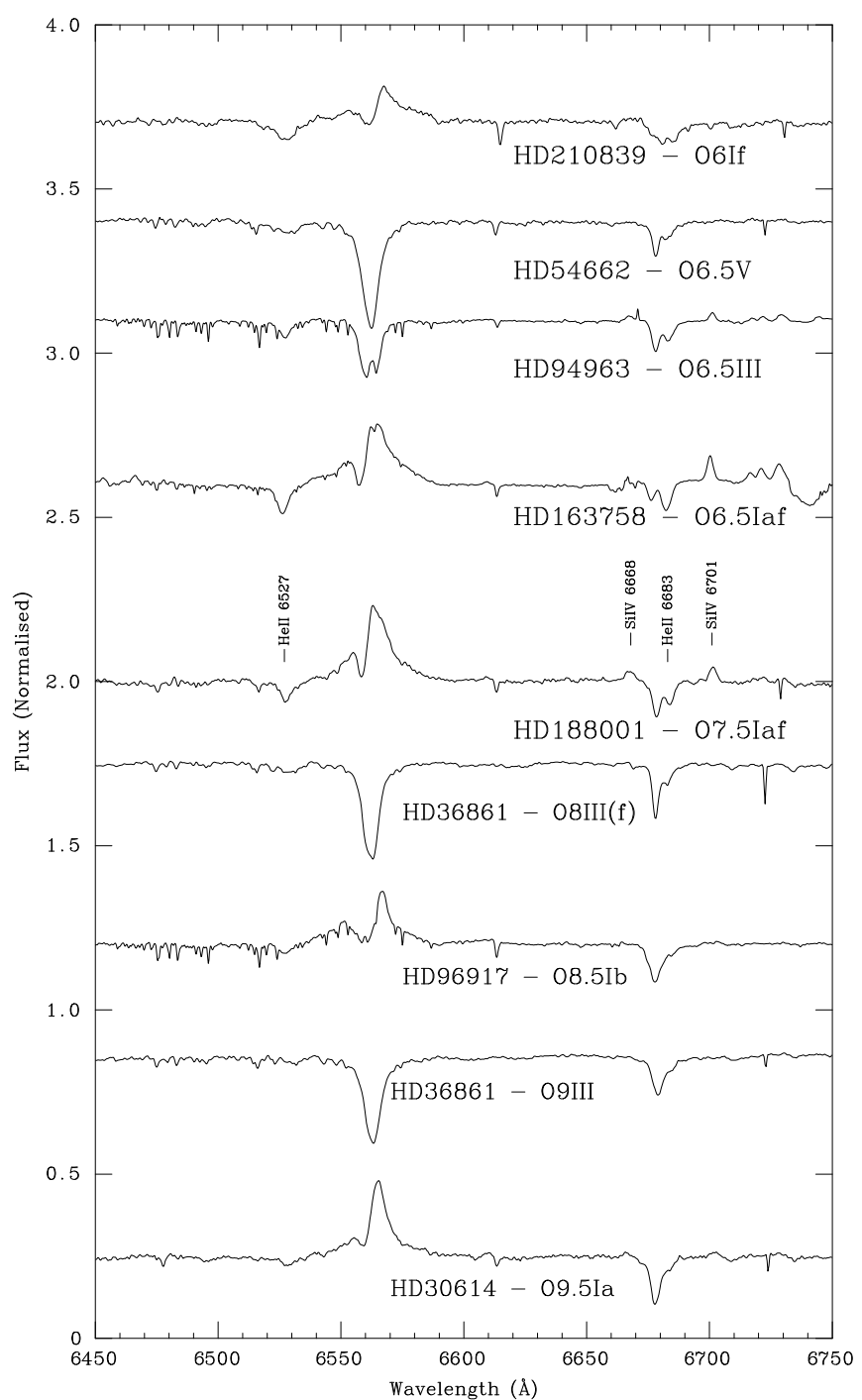

Fig. A.4. Spectral features seen around $\mathrm{H} \alpha$ in O-type stars. The spectra are drawn from several sources and have quite different resolutions. The He I $6678 \AA$ Aine has not been marked to prevent confusion.

in B1 supergiants at moderate resolution, but quickly grow in strength with advancing spectral type. They are seen in dwarfs from B2-3, depending on rotational velocity.

The C II 6578, $6582 \AA$ doublet is generally not seen in MS B-type stars, where it is very weak and easily masked by high rotation or low SNR. It is present in B2-B5 stars of moderate luminosity and in most B-type supergiants (Walborn 1980). As it is clearly seen in many luminosity class IV stars, we suspect that it may be seen in MS stars with very low $v_{\text {rot }}$. At high SNR, it is weakly visible in B0.5 supergiants. It clearly appears in B0.7 supergiants and its strength peaks around B3 (Lennon et al. 1993). The lines are visible until B8, and also in some B9 supergiants, with a clear dependance on luminosity. As seen in Fig. A.5, their strength is not monotonically correlated to spectral type amongst luminosity class Ia supergiants. There seems to be some degree of anticorrelation between the strength of these features and those of N II, suggesting that differences in $\mathrm{CNO}$ abundances play a role in their relative strength, as is seen in features further to the blue (Walborn \& Howarth 2000).

There are two N II lines in the range showed in Fig. A.5, which are only visible in supergiants. N II $6482 \AA$ appears 
Table A.1. Summary of features useful for spectral classification between O4 and B9 in the 6300-9000 A range.

\begin{tabular}{|c|c|c|c|c|}
\hline Feature & From & To & Maximum & Comments \\
\hline Paschen lines & $\mathrm{O} 4$ & B9 & $>\mathrm{B} 9$ & $\begin{array}{l}\text { Number of lines seen depends on temperature and luminosity } \\
\text { Shape of lines correlates with luminosity }\end{array}$ \\
\hline He I 6678, $7065 \AA$ & $\mathrm{O} 4$ & $\sim \mathrm{B} 7 \mathrm{~V}, \mathrm{~B} 9 \mathrm{I}$ & B2 & \\
\hline He I 8847, 8777, $8583 \AA$ & O8-9 & B3 V, B6 I & B2 & strength depends on luminosity \\
\hline He II $6683 \AA$ & $\mathrm{O} 4$ & O7 V, O9 I & $\mathrm{O} 4$ & blended with He I $6678 \AA$ \\
\hline He II $8283 \AA$ & $\mathrm{O} 4$ & O9 I-V & $\mathrm{O} 4$ & inside telluric band \\
\hline C III $8500 \AA$ & $\sim 06$ & B0.5 & O9 & lower limit not well determined \\
\hline C II 6578, $6582 \AA$ & В0.7 & B6 III, B8 I & B3 & rarely seen in dwarfs \\
\hline C II 7231, $7236 \AA$ & O9.7 I & B2 I & B1 & $\begin{array}{l}\text { emission features only in luminous SGs } \\
\text { inside telluric band }\end{array}$ \\
\hline O I $8446 \AA$ & B2 V B1.5 I & B9 & $>\mathrm{B} 9$ & strong dependence on luminosity \\
\hline O I $7774 \AA$ & B3 V B1 I & B9 & $>\mathrm{B} 9$ & strong dependence on luminosity \\
\hline Si II 6347, $6371 \AA$ & B2 I & B9 & B8 & tabulated by Lennon et al. (1993) in SGs \\
\hline Si IV 6668, $6701 \AA$ & - & O9 & $?$ & emission lines in SGs; lower limit not determined \\
\hline N I 8680-86, 8703-18 ̊̊ & B3 I-II & B9 I-II & $>\mathrm{B} 9$ & not seen in III-V \\
\hline N II 6482, $6610 \AA$ & B1 I & B3 I & B2 & only strong in Ia SGs \\
\hline Ne I $6402 \AA$ & B1 I & B9 I & B4-5 & tabulated by Lennon et al. (1993) in SGs \\
\hline
\end{tabular}

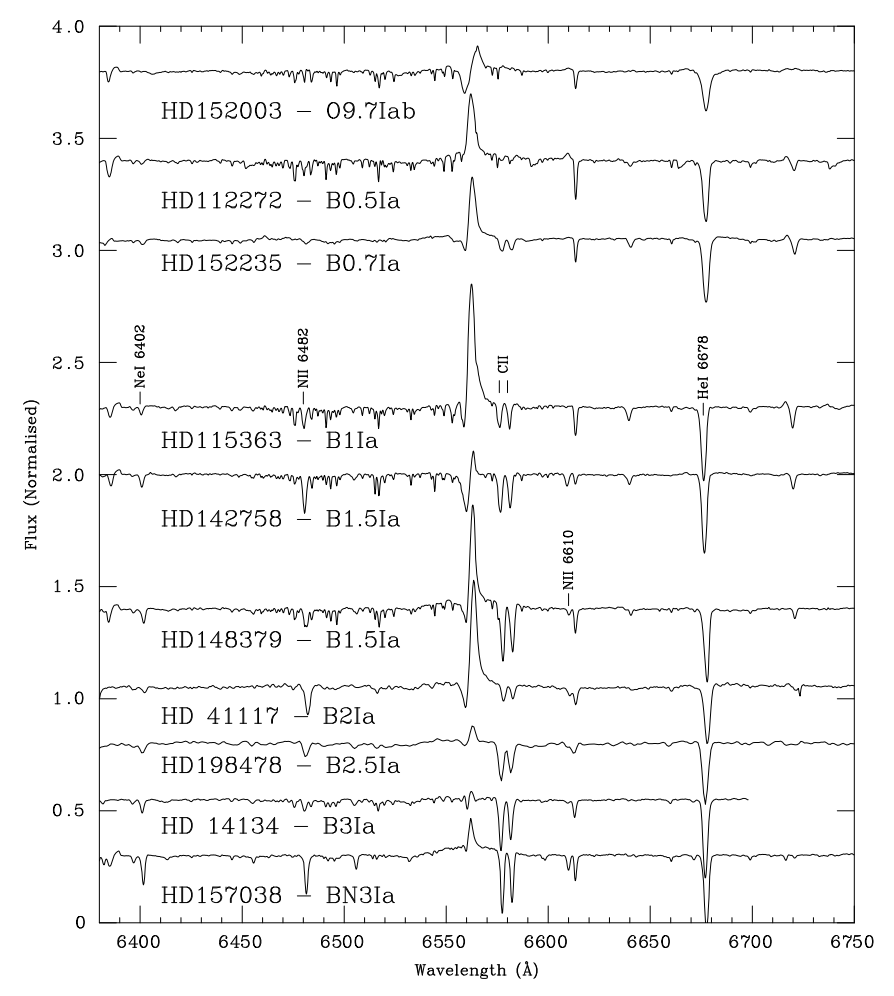

Fig. A.5. Spectral features seen around $\mathrm{H} \alpha$ in B-type supergiants stars. The spectra are drawn from several sources and have quite different resolutions. Spectra from the UVES POP show strong atmospheric features. The correlation between the intensity of the C II $6578,6582 \AA$ doublet and spectral type is loose because of $\mathrm{CNO}$ anomalies, as proved by the strong anticorrelation with the strength of $\mathrm{N}$ II lines at a given spectral type.

weakly in B1 supergiants, is rather strong at B2 and becomes weak again at B3, disappearing at later spectral types. Its dependence on temperature is very similar to that of $\mathrm{N}$ II lines in the blue range, such as N II $4601 \AA$, tabulated by Lennon et al. (1993). N II $6610 \AA$ is rather weaker, and has the same temperature dependence. As shown by Lennon (1993), these two lines are only prominent in B2 Ia supergiants. However, $\mathrm{N}$-enhanced stars of similar spectral types may also show relatively strong features (Fig. A.5). Another N II line behaving similarly is N II $6380 \AA$ (Walborn 1980).

The Ne I $6402 \AA$ line is seen in several of the spectra. Its behaviour was tabulated by Lennon et al. (1993). The line is visible from B1 to B9, and strongest at B4-5, but it does not seen to depend on luminosity subclass amongst supergiants. A weaker line, Ne I $6506 \AA$, is seen in the spectrum of HR Car, which is classified B2 (Nota et al. 1997). The Si II $6347 \AA$ line was also tabulated by Lennon et al. (1993). It is hardly visible at B2, but increases its strength very quickly, becoming prominent at B3 and growing in strength towards later spectral types. The neighbouring Si II $6371 \AA$ A behaves similarly. Features further to the blue are discussed by Walborn (1980).

\section{A.4. Summary and outlook}

The results presented here show that classification of early-type stars is possible using only red spectra. A summary of features useful for classification is presented in Table A.1. The classifications are not as accurate as those using blue spectra, but likely comparable to classifications based on $K$-band spectra. Combination of red spectra with near-IR spectra offers good prospects to classify rather accurately obscured early-type stars. The limited spectral range offered by GAIA does not allow accurate spectral classification, but the situation is not as desperate as suggested by Munari \& Tomasella (1999). As seen in Fig. 6, even O-type stars can be approximately classified in that range, at high resolution and signal-to-noise ratio.

Further improvement on classification and a more thorough understanding of some of the issues raised here may be obtained by a systematic study of MK standards and stars with accurate spectral types.

\section{References}

Abt, H. A., Levato, H., \& Grosso, M. 2002, ApJ, 573, 359

Andrillat, Y., Jaschek, C., \& Jaschek, M. 1988, A\&AS, 112, 475

Arellano Ferro, A., Giridhar, S., \& Rojo Arellano, E. 2003, RMxAA, 39, 3

Bagnulo, S., Cabanac, R., Jehin, E., et al. 2003, Messenger, 114, 10 
Bonanos, A. Z. 2007, AJ, 133, 2696

Brandner, W., Clark, J. S., Stolte, A., et al. 2008, A\&A, 478, 137

Caron, G., Moffat, A. F. J., St-Louis, N., et al. 2003, AJ, 126, 1415

Clark, J. S., \& Negueruela, I. 2004, A\&A, 413, L15

Clark, J. S., Negueruela, I., Crowther, P. A., \& Goodwin, S. P. 2005, A\&A, 434, 949 (C05)

Clark, J. S., Muno, M. P., Negueruela, I., et al. 2008, A\&A, 477, 147

Clark, J. S., Ritchie, B. W., \& Negueruela, I. 2010, A\&A, 514, A87

Cenarro, A. J., Cardiel, N., Gorgas, J., et al. 2001, MNRAS, 326, 959

Cox, N. L. J., Kaper, L., Foing, B. H., et al. 2005, A\&A, 438, 187

Crowther, P. A., Hadfield, L. J., Clark, J. S., et al. 2006a, MNRAS, 372, 1407

Crowther, P. A., Lennon, D. J., \& Walborn, N. R. 2006b, A\&A, 446, 279

Davies, B., Oudmaijer, R. D., \& Vink, J. S. 2005, A\&A, 439, 1107

Dougherty, S. M., Clark, J. S., Negueruela, I., et al. 2010, A\&A, 58, A54

Draper, P. W., Taylor, M., \& Allan, A. 2000, Starlink User Note 139.12, R.A.L.

Eldridge, J. J., Izzard, R. G., \& Tout, C. A. 2008, MNRAS, 384, 1109

van Genderen, A. M. 2001, A\&A, 366, 508

Howarth, I., Murray, J., Mills, D., \& Berry, D. S. 1998, Starlink User Note 50.21, R.A.L.

Humphreys, R. M., \& McElroy, D. B. 1984, ApJ, 284, 565

Humphreys, R. M., \& Davidson, K. 1994, PASP, 106, 1025

Kothes, R., \& Dougherty, S. M. 2007, A\&A, 468, 993

Le Borgne, J.-F., Bruzual, G., Pelló, R., et al. 2003, A\&A, 402, 433

Leitherer, C. 1988, ApJ, 326, 356

Lennon, D. J. 1993, Space Sci. Rev. 66, 127

Lennon, D. J., Dufton, P. L., \& Fitzsimmons, A. 1993, A\&AS, 97, 595

Markova, N., \& Valchev, T. 2000, A\&A, 363, 995

Markova, N., \& Puls, J. 2008, A\&A, 478, 823

Martins, F., Schaerer, D., \& Hillier, J. 2005, A\&A, 436, 1049

Massey, P. 2003, ARA\&A, 41, 15

McErlean, N. D., Lennon, D. J., \& Dufton, P. L. 1999, A\&A, 349, 553

Mengel, S., \& Tacconi-Garman, L. E. 2009, Ap\&SS, 324, 321

Meynet, G., \& Maeder, A. 2000, A\&A, 361, 101
Morel, T., Marchenko, S. V., Pati, A. K., et al. 2004, MNRAS, 351, 552

Munari, U., \& Tomasella, L. 1999, A\&AS, 137, 521

Munari, U., \& Zwitter, T. 1997, A\&A, 318, 269

Munari, U., Tomasella, L., Fiorucci, M., et al. 2008, A\&A, 488, 969

Muno, M. P., Law, C., Clark, J. S., et al. 2006, ApJ, 650, 203

Naylor, T. 2009, MNRAS, 399, 432

Negueruela, I., \& Clark, J. S. 2005, A\&A, 436, 541

Nota, A., Smith, L., Pasquali, A., et al. 1997, ApJ, 486, 338

Rieke, G. H., \& Lebofsky, M. J. 1985, ApJ, 288, 618

Ritchie, B. W., Clark, J. S., Negueruela, I., \& Crowther, P. A. 2009a, A\&A, 507, 1585

Ritchie, B. W., Clark, J. S., Negueruela, I., \& Najarro, F. 2009b, A\&A, 507, 1597

Salasnich, B., Bressan, A., \& Chiosi, C. 1999, A\&A, 342, 131

Sánchez-Blázquez, P., Peletier, R. F., Jiménez-Vicente, J., et al. 2006, MNRAS, 371,703

Shortridge, K., Meyerdicks, H., Currie, M., et al. 1997, Starlink User Note 86.15, R.A.L.

Smith, N., Vink, J. S., \& de Koter, A. 2004, ApJ, 615, 475

Thaller, M. L. 1997, ApJ, 487, 380

Valdes, F., Gupta, R., Rose, J. A., et al. 2004, ApJS, 152, 251

van der Hucht, K., Herrero, A., \& Esteban, C. 2003, A Massive Star Odyssey: From Main Sequence to Supernova, Proc. IAU Symp., 212, held 24-28 June 2001 in Lanzarote, Canary Islands, Spain (San Francisco: ASP) van Helden, R. 1972, A\&A, 19, 388

Vanbeveren, D., Van Bever, J., \& Belkus, H. 2007, ApJ, 662, L107

Walborn, N. R. 1971, ApJS, 23, 257

Walborn, N. R. 1980, ApJS, 44, 535

Walborn, N. R., \& Fitzpatrick, E. L. 1990, PASP, 102, 379

Walborn, N. R., \& Howarth, I. D. 2000, PASP, 112, 1446

Walborn, N. R., Howarth, I. D., Lennon, D. J., et al. 2002, AJ, 123, 2754

Wegner, W. 1994, MNRAS, 270, 229

Westerlund, B. E. 1987, A\&AS, 70, 311

Zinnecker, H., \& Yorke, H. W. 2007, ARA\&A, 45, 481 